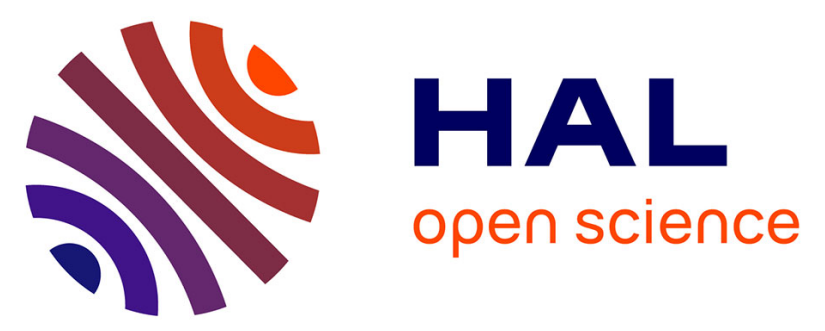

\title{
Influence of Coriolis Force Upon Bottom Boundary Layers in a Large-Scale Gravity Current Experiment: Implications for Evolution of Sinuous Deep-Water Channel Systems
}

S Davarpanah Jazi, M G Wells, J Peakall, R M Dorrell, R E Thomas, G M

Keevil, S E Darby, J. Sommeria, S Viboud, T Valran

\section{To cite this version:}

S Davarpanah Jazi, M G Wells, J Peakall, R M Dorrell, R E Thomas, et al.. Influence of Coriolis Force Upon Bottom Boundary Layers in a Large-Scale Gravity Current Experiment: Implications for Evolution of Sinuous Deep-Water Channel Systems. Journal of Geophysical Research. Oceans, 2020, 125 (3), pp.e2019JC015284. 10.1029/2019jc015284 . hal-03103151

\section{HAL Id: hal-03103151 https://hal.science/hal-03103151}

Submitted on 7 Jan 2021

HAL is a multi-disciplinary open access archive for the deposit and dissemination of scientific research documents, whether they are published or not. The documents may come from teaching and research institutions in France or abroad, or from public or private research centers.
L'archive ouverte pluridisciplinaire HAL, est destinée au dépôt et à la diffusion de documents scientifiques de niveau recherche, publiés ou non, émanant des établissements d'enseignement et de recherche français ou étrangers, des laboratoires publics ou privés. 


\author{
RESEARCH ARTICLE \\ 10.1029/2019JC015284 \\ Key Points: \\ - A large 13-m rotating flume is used \\ to study Ekman boundary layers \\ beneath a highly turbulent gravity \\ current in a sinuous channel \\ - The cross-stream flow at bed can \\ change direction and flow towards \\ inner bend if Coriolis force opposes \\ and exceeds the centrifugal force \\ - We discuss the potential \\ implications of Coriolis force upon \\ the development of high-latitude \\ channel levee systems on the ocean \\ floor
}

Supporting Information:

- Supporting Information S1

- Movie S1

- Movie S2

Correspondence to:

M. G. Wells,

wells@utsc.utoronto.ca

\section{Citation:}

Davarpanah Jazi, S., Wells, M. G., Peakall, J., Dorrell, R. M., Thomas, R. E., Keevil, G. M., et al. (2020). Influence of Coriolis force upon bottom boundary layers in a large-scale gravity current experiment: Implications for evolution of sinuous deep-water channel systems. Journal of Geophysical Research: Oceans, 125, e2019JC015284. https://doi.org/10.1029/2019JC015284

Received 13 MAY 2019 Accepted 1 FEB 2020

Accepted article online 10 FEB 2020

(C)2020. American Geophysical Union. All Rights Reserved.

\section{Influence of Coriolis Force Upon Bottom Boundary Layers in a Large-Scale Gravity Current Experiment: Implications for Evolution of Sinuous Deep-Water Channel Systems}

\author{
S. Davarpanah Jazi' ${ }^{1}$ D, M. G. Wells ${ }^{1}$ (D) J. Peakall ${ }^{2}$ (D) R. M. Dorrell ${ }^{3}$ (D) R. E. Thomas ${ }^{3}$ (D),

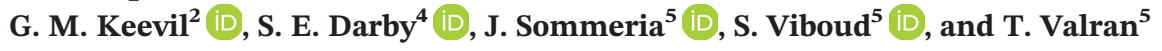 \\ ${ }^{1}$ Department of Physical and Environmental Sciences, University of Toronto Scarborough, Toronto, Ontario, Canada, \\ ${ }^{2}$ School of Earth \& Environment, University of Leeds, Leeds, UK, ${ }^{3}$ Energy and Environment Institute, University of Hull, \\ Hull, UK, ${ }^{4}$ School of Geography and Environmental Sciences, University of Southampton, Southampton, UK, \\ ${ }^{5}$ Laboratoire des Écoulements Géophysiques et Industriels (LEGI), Grenoble, France
}

\begin{abstract}
Oceanic density currents in many deep-water channels are strongly influenced by the Coriolis force. The dynamics of the bottom boundary layer in large geostrophic flows and low Rossby number turbidity currents are very important for determining the erosion and deposition of sediment in channelized contourite currents and many large-scale turbidity currents. However, these bottom boundary layers are notoriously difficult to resolve with oceanic field measurements or in previous small-scale rotating laboratory experiments. We present results from a large, $13-\mathrm{m}$ diameter, rotating laboratory platform that is able to achieve both stratified and highly turbulent flows in regimes where the rotation is sufficiently rapid that the Coriolis force can potentially dominate. By resolving the dynamics of the turbulent bottom boundary in straight and sinuous channel sections, we find that the Coriolis force can overcome centrifugal force to switch the direction of near-bed flows in channel bends. This occurs for positive Rossby numbers less than +0.8 , defined as $R o_{R}=\langle\bar{U}>/ R f$, where $\langle\bar{U}>$ is the depth and time-averaged velocity, $R$ is the radius of channel curvature, and $f$ is the Coriolis parameter. Density and velocity fields decoupled in channel bends, with the densest fluid of the gravity current being deflected to the outer bend of the channel by the centrifugal force, while the location of velocity maximum shifted with the Coriolis force, leading to asymmetries between left- and right-turning bends. These observations of Coriolis effects on gravity currents are synthesized into a model of how sedimentary structures might evolve in sinuous turbidity current channels at various latitudes.
\end{abstract}

Plain Language Summary Many of the largest currents in the oceans depths are dense, gravity-driven, flows that pour down deep-water channels. These large gravity currents include dense overflows, as well as sediment-laden turbidity currents and contourite flows. The dynamics of these gravity currents can be strongly affected by the Coriolis force. This study examines the effect of the Coriolis force on the flow structure of oceanic gravity currents flowing through straight and sinuous channels. A set of 22 experiments were carried out on the world's largest rotating experimental facility, the LEGI Coriolis platform in Grenoble, France. Our detailed measurements of velocity and internal density structure imply that at higher latitudes Coriolis force dominates and changes the direction of the flow near the bed leading to differences between flows going around a left-turning versus a right-turning bend. Our observations are relevant to the large deep-water channels formed on the ocean floor by successive turbidity currents. Near the Equator these channels tend to be noticeably sinuous; however, recent studies have shown that this sinuosity decreases with latitude. One possibility is that latitudinal variations in the Coriolis force may influence the evolution of these channels through changing near-bed patterns of erosion and deposition.

\section{Introduction}

Oceanic density currents drain through deep-water channels. Examples of oceanic density currents include dense water flows from marginal seas (Ivanov et al., 2004) and large-scale overflows (Legg et al., 2009). In addition to these well-known thermohaline flows, there are a class of geologically important density currents, namely, contourite currents and turbidity currents, which are important for the distribution of sediment, carbon, heat, and pollutant fluxes in the oceans (Peakall \& Sumner, 2015; Rebesco et al., 2014; 
Wynn et al., 2007). Submarine channels are very large geological features that are formed by the passage of successive turbidity currents over thousands of years (Maslin et al., 2006; Piper \& Deptuck, 1997). At high latitudes, such channels are known to be strongly influenced by the Coriolis force (e.g., Akhmetzhanov et al., 2007; Hesse et al., 1987), in marked contrast to rivers, where the Coriolis force is always small (Cossu et al., 2010, 2015; Cossu \& Wells, 2013; Komar, 1969). Submarine channels have recently been shown to exhibit a global variation in channel sinuosity, with highly sinuous channels in equatorial regions, progressively decreasing to near straight channels at latitudes higher than $50^{\circ}$, in complete contrast to rivers (Peakall et al., 2012). Explanations for this global sinuosity variation, most notably for larger channels, have been linked to Coriolis and rotational effects (Cossu \& Wells, 2013; Peakall et al., 2013; Wells \& Cossu, 2013; Cossu et al., 2015). However, experimental investigations of the influence of Coriolis on the fluid dynamics of these channels have largely been limited to velocity profiles and isolated low-resolution two-dimensional cross-sectional velocity fields. These experiments have been too small to assess the development of Ekman boundary layers (Cossu et al., 2010, 2015; Wells \& Cossu, 2013) that would potentially dominate the flows near the beds of these channels, where sediment erosion and deposition takes place. Most previous laboratory experiments investigating Coriolis effects on gravity currents were at relatively low Reynolds numbers in shallow flows (Cenedese et al., 2004; Cenedese \& Adduce, 2008; Cossu et al., 2010, 2015; Cossu \& Wells, 2010; Wåhlin et al., 2008; Wells, 2009; Wells \& Cossu, 2013), making it difficult to measure properties in these laminar or nearly laminar boundary layers. Hence, in order to understand how the near-bed turbulence levels and secondary circulation patterns of turbulent gravity currents potentially vary as a function of latitude, it is important to conduct gravity current experiments in deeper flows at large Reynolds numbers.

The Coriolis force acts at right angles to the direction of the flow and can change the dynamics of gravity currents flowing in a channel in several fundamental ways. First, the upper density interface of a gravity current will be deflected laterally; in a straight channel this interface will be deflected until the flow reaches a geostrophic balance between the pressure gradient and Coriolis forces (Cossu et al., 2015; Darelius \& Wåhlin, 2007; Komar, 1969). In such a case, when flows come into a geostrophic balance, the bulk speed of a gravity current can be substantially reduced (Cossu et al., 2010) with the speed approximately given by the "Nof velocity" (Wåhlin et al., 2008). Second, the lateral Coriolis force can induce substantial secondary flows even in gravity currents flowing in straight channels (Darelius, 2008; Johnson \& Ohlsen, 1994). In a straight channel, the Coriolis force tends to deflect the bulk of the flow to the right-hand side in the Northern Hemisphere and to the left in the Southern Hemisphere when looking downstream (Cossu et al., 2010), as depicted schematically in Figure 1a. On the other hand, when a current enters a curved channel (with a bend turning to the left) centrifugal and Coriolis forces join each other when looking downstream in the Northern Hemisphere, whereas the two forces oppose each other in the Southern Hemisphere (Figure 1b). For a gravity current flowing in a straight channel a Rossby number is defined in terms of the channel width $(W)$, and depth-and time averaged velocity $(\langle\bar{U}\rangle)$ where angle brackets denote a time-average and an overbar a depth-average, and Coriolis parameter $(f)$ as

$$
R o_{W}=\frac{<\bar{U}>}{f W}
$$

The circulation in a sinuous channel is a function of the effect of the centrifugal and Coriolis forces on the downstream velocity, which leads to a deflection of the flow and produces cross-stream velocities. To determine the dominating force, a Rossby number is instead defined in terms of the channel radius of curvature $(R)$ as

$$
R o_{R}=\frac{<\bar{U}>}{f R} .
$$

With these definitions the two Rossby numbers are related as $R o_{R}=R o_{W}(W / R)$. The Coriolis parameter is defined as twice the solid body rotation rate $(\Omega)$, i.e., $f=2 \Omega$. For an oceanographic flow on the (near-) spherical earth, it is the projection of the rotational vector that matters and so Coriolis varies with latitude $(\theta)$ as $f=2 \Omega \sin (\theta)$, where $\Omega=2 \pi$ /day for the Earth. We note that the radius $R$ has a sign indicating whether the Coriolis force acts either with or against the centrifugal force (Cossu \& Wells, 2010), so that for a given channel in the Northern Hemisphere, a bend turning to the left (looking downstream) has positive $R o_{R}$ 
a

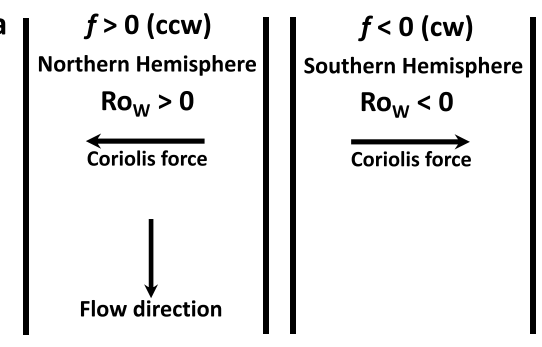
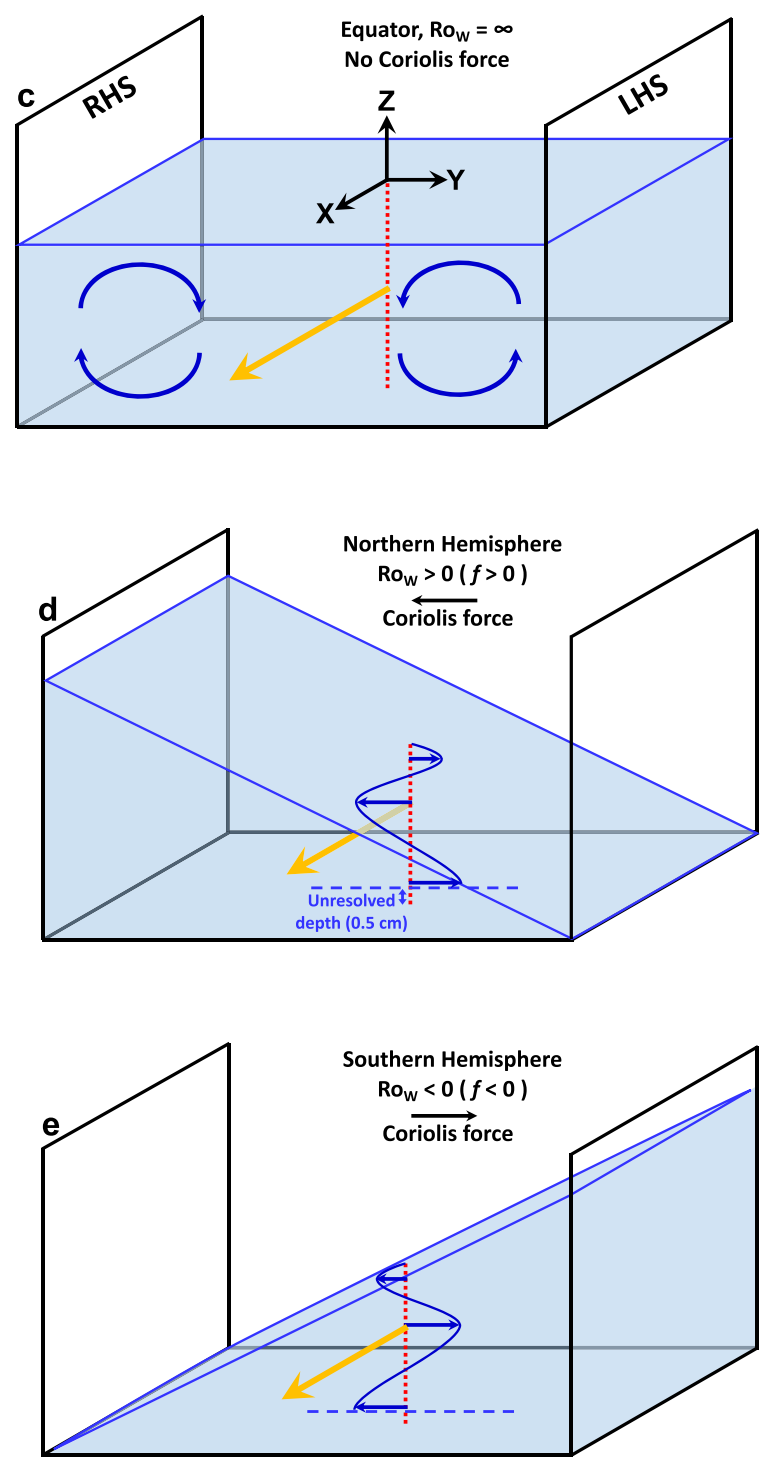
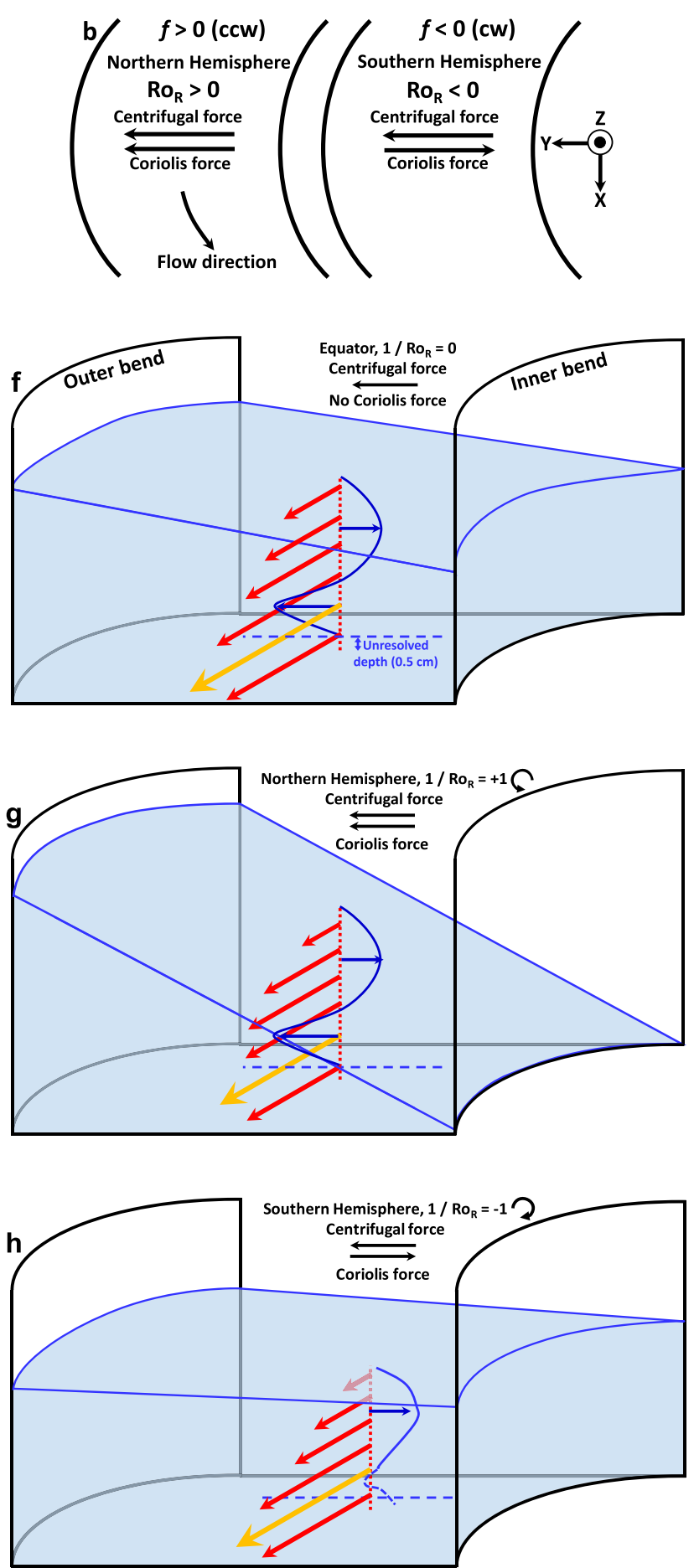

Figure 1. A schematic of the direction of the centrifugal and Coriolis forces in a channel in the Northern and Southern Hemispheres in (a) the straight section (position X1 in the experiments) and (b) the second bend apex (position X2 in the experiments). A schematic of the downstream and cross-stream velocities in a straight channel, with (c) no rotation, (d) Northern Hemisphere rotation, and (e) Southern Hemisphere rotation, based upon the data in Cossu et al. (2010). Schematics of the downstream and cross-stream velocities in a left-turning channel bend are sketched for the cases of (f) no rotation, (g) Northern Hemisphere rotation, and (h) Southern Hemisphere rotation, based upon the data from Cossu and Wells (2010). In (h) the dashed line represents the ambiguity in what happens in bottom $2.5 \mathrm{~cm}$. The red, yellow, and blue vectors show the downstream, maximum downstream, and cross-stream velocities, respectively. The basal blue dashed line represents the region below $0.5 \mathrm{~cm}$ height where flows in the bottom boundary layer could not be measured. Furthermore, even the lowermost measurements at the $0.5-\mathrm{cm}$ position were an average of the flow above and below this flow height and thus are unreliable (see text for details). 


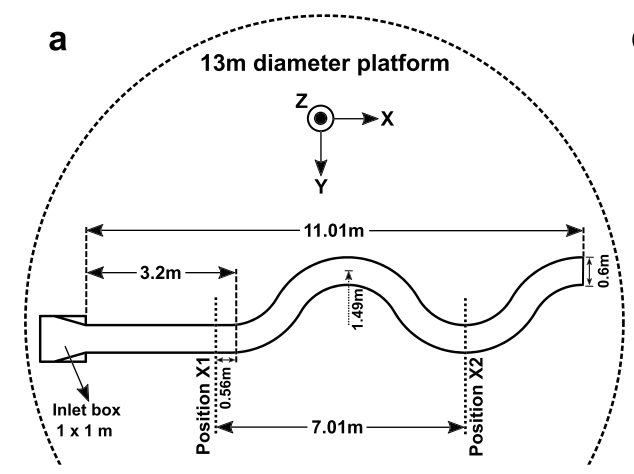

C RHS
(Outer wall)
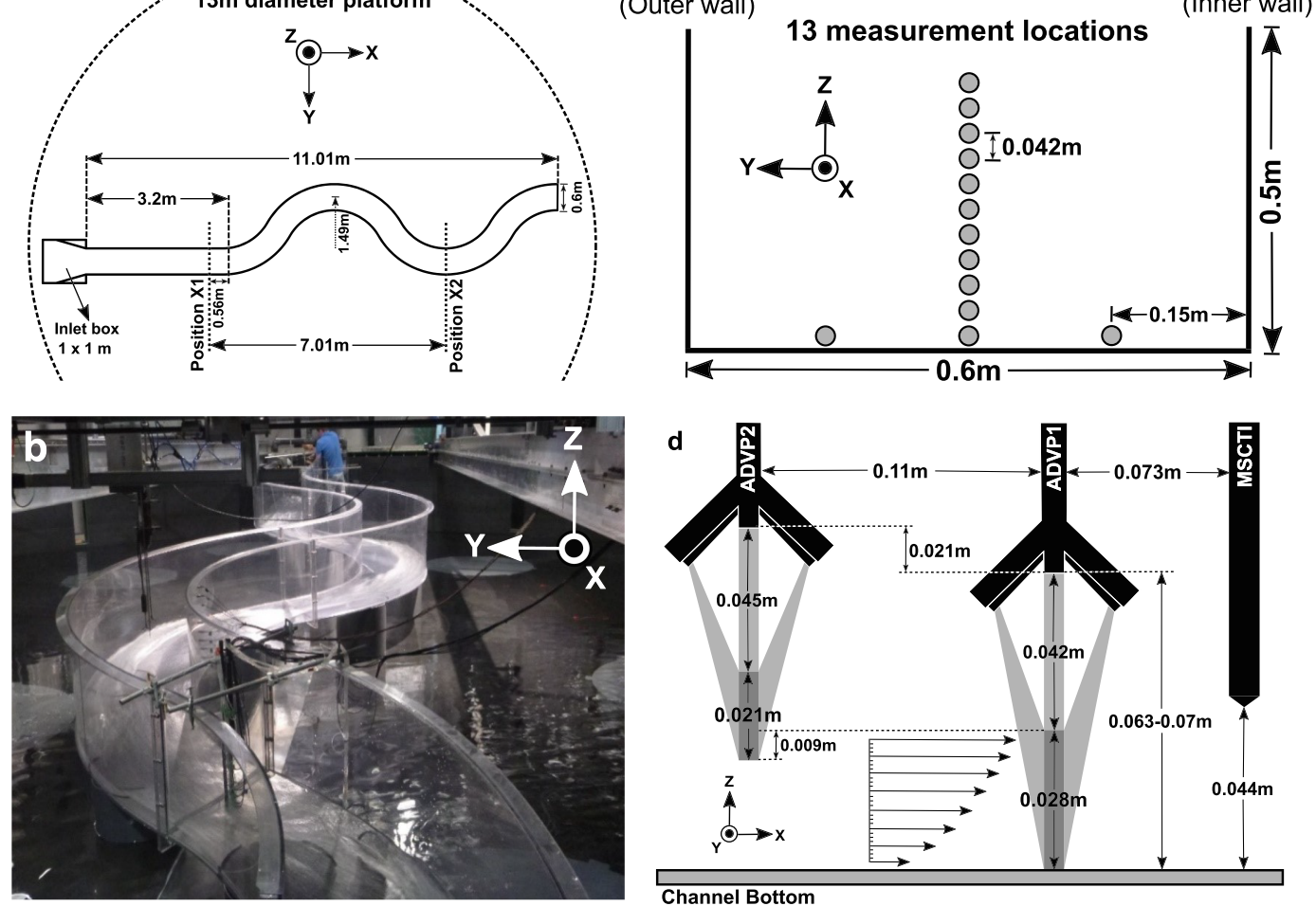

Figure 2. (a) Schematic of the channel used in the rotating gravity current experiments at the Coriolis facility at LEGI in Grenoble. The source at the inlet box (located on the left) supplied 5.7 to $5.9 \mathrm{~L} \mathrm{~s}^{-1}$ of saline fluid that flowed down the $3.4^{\circ}$ slope. Data were collected at two locations along the channel named position X1 and position X2. (b) A photo of the 11.01-m long Plexiglas channel with radius of curvature of $R=1.49 \mathrm{~m}$ located inside the rotating table, looking upstream. (c) The velocity measurement locations within the channel at two positions of X1 and X2. (d) The detailed position of the ADVPs and the MSCTI conductivity probe used in the experiments. The MSCTI probe was laterally offset by $0.055 \mathrm{~m}$ in the Y direction from the two ADVP units. The dark gray area shows the measurement volume in which the seeded particles reflect the transmitted sound pulse.

(Coriolis force acts in the same direction as centrifugal force), while a bend turning to the right has negative $R o_{R}$ (Coriolis force is in the opposite direction to the centrifugal force). Previous work by Cossu and Wells (2010) and Wells and Cossu (2013) documented how the tilt of the upper interface depends upon $R o_{R}$. In a sinuous channel, alternating bends will change the sign of the Rossby number, but $R o_{R}=-1$ is the specific case where the Coriolis and centrifugal forces are balanced. This lead Komar (1969) to predict that for this special case the upper interface of a gravity current would be parallel with Earth's surface as the density current flowed around a bend (assuming a bend of constant curvature).

The main effects of the Coriolis force upon the velocity structure of gravity currents when flowing in straight and sinuous submarine channels are summarized in Figure 1, based upon previous studies (Cossu et al., 2010; Cossu \& Wells, 2010). The coordinate system is introduced in Figures 1a and 1b, so that the centrifugal and Coriolis forces act in the same direction for left-turning channels in the Northern Hemisphere, i.e., having a positive $R o_{R}$. A summary of the mean flow structure of a density current in a straight channel is shown in Figures 1c-1e, which schematically summarizes the data of Cossu et al. (2010). The downstream velocity measurements of Cossu et al. (2010) included a single data point located $0.025 \mathrm{~m}$ above the bottom and at the center of a straight channel with a current thickness of approximately $0.06 \mathrm{~m}$. Cross-stream velocities were measured at six points (vertically spaced $0.01 \mathrm{~m}$ from each other), starting $0.005 \mathrm{~m}$ from the bottom. Figure $1 \mathrm{c}$, corresponding to their nonrotating case $\left(R o_{W}=\infty\right)$, displays the helicoidal flow that converges at the top and diverges at the bottom through two adjacent cells spinning in opposite directions. However, the flow is deflected to the right when it experiences positive rotation (Figure 1d). Cossu et al. (2010) report interior flows of up to $0.005 \mathrm{~m} \mathrm{~s}^{-1}$ surrounded by two return flows at the top and the bottom of the gravity current. They postulated that such return flows are influenced by Ekman dynamics, and crossstream velocities (as measured perpendicular to the channel, see Peakall \& Sumner, 2015, for a discussion of 
reference frames) can be of the order of $10 \%$ of the downstream velocities in these channels. Figure 1e corresponds to negative rotation rate, which basically mirrors the flow field displayed in Figure 1d as the rotation rate remains the same, but direction is reversed. Furthermore, for Rossby numbers smaller than two, Cossu et al. (2010) found a good agreement between their theoretical model that included Ekman boundary layer dynamics and velocity observations from a gravity current in a straight channel. Other experimental studies (Darelius, 2008; Davies et al., 2006; Johnson \& Ohlsen, 1994) and studies of gravity currents in nature (Fer et al., 2010; Johnson \& Sanford, 1992; Sherwin, 2010; Umlauf \& Arneborg, 2009a, 2009b) have reported the same behavior of flow fields and secondary circulation patterns as Cossu et al. (2010). Note that, due to the small scales of the flow in Cossu et al. (2010), they could not directly investigate the dynamics of the Ekman boundary layer as they calculated it to be thinner than $0.005 \mathrm{~m}$ (assuming a viscosity of $10^{-6} \mathrm{~m}^{2}$ $\mathrm{s}^{-1}$ ) and therefore not detectable by the Metflow Ultrasonic Doppler Velocity Profiler (UDVP) used in their experiments.

A schematic summary of the previous descriptions of how the Coriolis force deflects flows in a sinuous channel is shown in Figures 1f-1h. Figures $1 \mathrm{~g}$ and $1 \mathrm{~h}$ summarize the findings of Cossu and Wells (2010) and Cossu et al. (2015) for the influence of strong Coriolis effects, while the behavior of a supercritical current in a bend without rotation is illustrated in Figure 1f. Starting with the case of no Coriolis force (Figure 1f), the maximum centrifugal force occurs at the level of maximum downstream velocity, and the average sense of secondary flows is determined by the location of the velocity maximum, the degree of net cross-stream fluid movement associated with changes in superelevation, and the nature of changes in the cross-stream density distribution (Dorrell et al., 2013, 2018; Peakall \& Sumner, 2015). For supercritical flows, the strong mixing at the upper interface dominates the drag on the density current (Meiburg \& Kneller, 2010; Turner, 1973; Wells et al., 2010), which results in the downstream maximum velocity being quite close to the bottom of the current. The secondary flow is then in the direction of the outer bend at the bed and a return flow occurs at the surface (Dorrell et al., 2013; Keevil et al., 2006; Peakall \& Sumner, 2015; Serchi et al., 2011). The main secondary circulation in the nonrotating supercritical experiments of Cossu and Wells (2010) similarly shows basal flow directed towards the outer bank (Figure 4a of Cossu \& Wells, 2010). The case shown in Figure 1f is for a single flow cell; however, in some experimental and numerical cases multiple vertically stacked secondary flow cells are observed, with the basal flow in the lowermost secondary flow cell, typically oriented towards the inner bank (Dorrell et al., 2013, 2018; Imran et al., 2007; Islam \& Imran, 2008). A developing lower secondary cell with basal flow towards the inner bank was suggested in the experiments of Cossu and Wells (2010), however, as noted earlier the resolution in the basal measurements was limited. Studies by Keevil et al. (2006), Imran et al. (2007), and Dorrell et al. (2018) reported cross-stream velocities of order 10\% of the mean flow for nonrotating experiments. When the Coriolis force comes into effect and acts in the same direction as the centrifugal force $\left(R o_{R}>0\right)$, as indicated in Figure $1 \mathrm{~g}$, the structure of the flow is quite similar to the nonrotating case, with the major difference being a super elevation of the upper interface. While there may have been a small drop in cross-stream flow, the previous observations of Cossu and Wells (2010) were not able to resolve if the magnitude of cross-stream velocity changed significantly and also did not resolve the bottom boundary layer. On the other hand, when the Coriolis force opposes the centrifugal force (Figure $1 \mathrm{~h}$ ), secondary flow decreases and in some cases is almost absent (see Figures 3c and 3d of Cossu \& Wells, 2010). However, the estimate of the Ekman boundary layer thickness by Cossu and Wells (2010) is much smaller than $0.01 \mathrm{~m}$, indicating that it was not detected by their UDVP (indicated by a dashed line in Figure 1h). Note that the UDVP technique used in these shallow flows ( 6-cm depth; Cossu \& Wells, 2010; Cossu et al., 2010) had a varying spatial resolution across the channel (vertical sampling size of $\sim 0.15-0.75 \mathrm{~cm}$ across the channel), and given that the lowermost probe was positioned with the transducer center at $0.005 \mathrm{~m}$ above the floor, then this lowermost probe may have sampled the Ekman boundary layer, but if so would have averaged measurements from both the Ekman boundary layer and the overlying flow. Hence, it was difficult to predict how these flows would transport sediment in the bottom boundary layer.

The growth and evolution of sinuous submarine channels could be influenced by how the Coriolis force deflects the upper interface and internal density structure of a turbidity current. The potential effects on the upper interface are more obvious and were identified by Komar (1969) as a tendency for the Coriolis and centrifugal effects to work together in left-turning bends in the Northern Hemisphere, thereby 
producing higher super elevation of flows, and hence, higher channel banks, than right-turning bends. A less obvious effect of the Coriolis force is that it could also influence secondary and Ekman boundary layer flows at the bed of sinuous channels, driving different sediment transport regimes than nonrotating flows. This effect was investigated by Cossu et al. (2015), who conducted a series of experiments over an erodible bed and simulated high and low latitude situations by changing the magnitude of the Coriolis force. They found that deposition occurred on alternating inner bends of sinuous channel at low latitudes (Cossu et al., 2015), confirming earlier experimental and numerical work on nonrotating sinuous channel deposition (Amos et al., 2010; Darby \& Peakall, 2012; Peakall et al., 2007). Conversely, patterns of erosion and deposition changed significantly, and both were located on the same side of sinuous channel bends at high latitudes, where the Coriolis force dominates $\left(\left|1 / R o_{R}\right|>1\right)$. To further understand how the Coriolis force influences the evolution of these geological systems it requires a better understanding of the flow near the boundary, which will be related to the development of an Ekman boundary layer.

The classical descriptions of an idealized Ekman boundary layer require there to be no stratification, a flat bottom, no centrifugal force, and a constant viscosity, conditions that clearly do not hold in any real channelized gravity current at large oceanographic scales (Gong et al., 2017; Peakall \& Sumner, 2015). Ekman boundary layer theory can be modified to include how stratification, or a nonconstant turbulent diffusivity, might modify a deep flow over a flat boundary (summarized in Cushman-Roisin, 1994), as for flows in the atmosphere or ocean. However, there is no theory for how the Coriolis force influences the boundary layer flow beneath currents that are both very strongly stratified and have pronounced vertical gradients of horizontal velocity. There have only been a few studies looking at high Reynolds number Ekman boundary layers without stratification (Caldwell et al., 1972; Ferrero et al., 2005; Howroyd \& Slawson, 1975; Sous et al., 2013) or in lower Reynolds number gravity currents with stratification (Cossu et al., 2010; Cossu \& Wells, 2010; Darelius, 2008; Johnson \& Ohlsen, 1994). While field observations of turbulent Ekman boundary layers beneath large-scale gravity currents are very helpful (Johnson \& Ohlsen, 1994; Johnson \& Sanford, 1992; Muench et al., 2009; Peters \& Johns, 2006; Seim \& Fer, 2011), these are fairly limited in terms of covering a sufficient number of locations at different latitudes to yield a range of Ekman and Rossby numbers. Hence, in order to understand the potential role of Ekman boundary layers in driving erosional/depositional patterns in turbidity currents flowing in large sinuous channels, an important first step is to understand how secondary flows and turbulence magnitude change near the bed underneath a high Reynolds number gravity current over a range of Coriolis parameter values representing flows near the Equator and flows at high latitudes.

In the current study, we focus on the effect of the Coriolis force on erosion at the bottom of deep-water channels and the shear stress that is caused by these large-scale currents. Our objective is to measure detailed velocity and turbulence distributions within channelized gravity currents, as a function of the Coriolis force, concentrating on the bottom boundary layer. This bottom boundary layer has important implications for basal shear stress distributions, the direction of sediment transport in the bottom boundary layer, and hence, the evolution of deep-water channels. The data on the distribution of turbulence will then be applied to provide an analysis of secondary flow and associated turbulence around bends, for the first time. We conclude with an assessment of how channelized flows alter as a function of Rossby number and therefore latitude, exploring the implications of how this effect might shift the locations of erosion and deposition within channels and hence, the evolution of channel sinuosity.

\section{Materials and Methods}

\subsection{Parameters and Definitions}

The dynamics of rotating gravity currents can be described in terms of four key dimensionless variables; the vigor of turbulence can be described by a Reynolds number $(R e)$, the stabilizing effects of stratification are described by a bulk Froude number $(F r)$, the influence of Coriolis force can be defined by two Rossby numbers in terms of channel width $\left(R o_{W}\right)$ or channel curvature $\left(R o_{R}\right)$, and the importance of the rotation within the well-mixed bottom boundary layer can be described by an Ekman number (Ek). Using these dimensionless parameters allows a meaningful extrapolation of the dynamics of our laboratory experiments to field-scale settings, and a useful comparison between various laboratory experiments, as shown in the supporting information, Table S1. The bulk Reynolds number of the flow $(R e)$ is defined as 


$$
R e=\frac{<\bar{U}>H}{\nu}
$$

where $\langle\bar{U}>$ is the velocity time-averaged over the experiment and depth-averaged over the depth of the gravity current $(H)$ and $v$ is the kinematic viscosity of water (which at average laboratory conditions of $21^{\circ} \mathrm{C}$ is $\nu=10^{-6} \mathrm{~m}^{2} \mathrm{~s}^{-1}$ ). $H$ can be defined in several ways, such as identifying a depth of velocity reversal (Dorrell et al., 2016; Wei et al., 2013) or various integrals of momentum and buoyancy (Ellison \& Turner, 1959; Sequeiros, 2012; Wells et al., 2010). While these integral methods are effective at describing low Froude number flows with sharp interfaces, they do not work as well for turbulent flows with diffuse interfaces and often predict flow depths that are below a significant portion of the density or velocity gradients. Hence, for pragmatic reasons we define $H$ as the depth of the flow from the bottom of the channel to the upper point where the downstream velocity is $5 \%$ of the maximum downstream velocity $\left(U(z)=0.05 U_{\max }\right)$. This definition of $H$ gives very similar results to other methods but is more straightforward to calculate when the diffuse density interface is laterally tilted, as occurs in the present experiments.

The densimetric Froude number for the flow is defined as

$$
F r=\frac{<\bar{U}>}{\sqrt{g^{\prime} H}},
$$

where the reduced gravity $g^{\prime}$ is defined as $g^{\prime}=g\left(\bar{\rho}-\rho_{O}\right) / \rho_{O}$ in terms of the difference between depth-averaged density of the fluid $(\bar{\rho})$ within the depth $H$ and the density of the ambient fresh water above the gravity current $\left(\rho_{O}\right)$. The density of the ambient fluid ranged between 998.3 and $998.8 \mathrm{~kg} \mathrm{~m}^{-3}$ and was measured for every experiment using an Anton Paar DMA 35 portable densitometer with a manufacturer-stated accuracy of $\pm 1 \mathrm{~kg} \mathrm{~m}^{-3}$ and a resolution of $0.1 \mathrm{~kg} \mathrm{~m}^{-3}$.

The degree to which Coriolis or frictional forces dominate within a well-mixed bottom boundary layer can be expressed by a turbulent Ekman number (Ek), which can be defined as (Arneborg et al., 2007; CushmanRoisin, 1994; Perlin et al., 2007)

$$
E k=\frac{U^{*}}{f h},
$$

where $U^{*}$ is the near-bed shear velocity and following the study by Johnson and Sanford (1992) on Ekman layers in the Faroe Bank channel outflow, the relevant length-scale $h$ is the elevation of the maximum downstream velocity from the bed. The near-bed shear velocity $\left(U^{*}\right)$, in the region below the velocity maximum of depth $h$, was calculated in experiments from the vector sum of downstream and cross-stream Reynolds stresses. In this paper we will report near-bed means of $U^{*}$ over the depth $h$. This region near the bed is also most likely to be well mixed. Above the depth of the velocity maximum, the gravity current is strongly stratified and hence, would not be expected to contain a classical Ekman boundary layer, which was derived for a uniform background flow in a homogenous fluid (Cushman-Roisin, 1994). Within the well-mixed layer, frictional force dominates when $E k>>1$, whereas Coriolis force dominates when $E k$ $<<1$ (Cushman-Roisin, 1994). From the definition of Ekman number in equation (5), $E k=\mathrm{O}(1)$ would imply that the flow dynamics in the well-mixed near-bed boundary layer is in a balance between friction and Coriolis forces. The predicted depth of an Ekman boundary $(d)$ can be approximated by setting Ek= 1 and rearranging equation (5) to give a length-scale $d \sim U^{*} / f$. Therefore, the predicted depth of a theoretical Ekman boundary layer $(d)$ decreases as $f$ increases, implying that the process of transferring momentum vertically becomes more efficient as rotation increases. Observations of temperature and velocity profiles made by Mercado and Van Leer (1976) from well-mixed boundaries on the southwest Florida continental shelf suggest that the Ekman layer depth, $d$, scales as (Mercado \& Van Leer, 1976; Perlin et al., 2007)

$$
d=0.4 U^{*} / f .
$$

Hence, using equations (5) and (6), the ratio of the theoretical Ekman boundary layer thickness $d$, to the depth $h$ of the well-mixed layer below the velocity maximum of a gravity current, should scale as 


$$
d / h=0.4 E k
$$

This scaling implies that there would only be enough depth for a classical Ekman spiral to be accommodated in the well-mixed boundary layer of a gravity current for cases of $E k<2.5$, as only in these cases would $d<h$ (see also Darelius, 2008). Conversely, the predicted Ekman boundary layer would be thicker than the well-mixed boundary layer for cases where $E k>2.5$ (where $d>h$ ) and hence, would be strongly influenced by the overlying stratification. Therefore, while the previous experiments of Cossu and Wells (2010) and Cossu et al. (2010) were somewhat turbulent, there would have been a stratification-modified Ekman boundary layer in their lower layers in the cases where $E k>2.5$. While there are some studies on a stratification-modified Ekman boundary layer (Garrett et al., 1993; Price \& Sundermeyer, 1999; Taylor \& Sarkar, 2008), there is no simple theory to describe velocity structure beneath a turbulent and stratified gravity current. This provides further justification of the need for present experiments. We note that the interpretation of Ekman in equation (7) is essentially the same as by Arneborg et al. (2007) and Cenedese et al. (2004), who also interpret the Ekman number as defining the ratio of a relevant vertical length-scale of a gravity current to the thickness of an Ekman boundary layer. We further note that the turbulent Ekman number in equation (5) can be derived from the standard definition of a laminar and viscous Ekman boundary layer by assuming that a constant turbulent viscosity can be defined from shear velocity as $v_{t u r b} \sim U^{*} d$ (Cushman-Roisin, 1994). Substitution of this expression for viscosity into the standard definition for a laminar Ekman number (where $E k=v / f d^{2}$; Cushman-Roisin, 1994) then leads to equation (5).

\subsection{Experimental Setup}

The experiments were conducted at the LEGI Coriolis Platform in Grenoble, France. This facility consists of a 13-m diameter, 1.2-m deep circular pool, which is built on a 16-m diameter concrete foundation. This setup can spin at different clockwise or counterclockwise spinning rates to simulate the Coriolis forces representative of Southern and Northern Hemispheres of the Earth. An 11-m long channel model was positioned within the pool. The 0.6-m wide and 0.5-m deep transparent acrylic channel model consisted of an initial tapered inlet section with a honeycomb baffle for flow straightening and turbulence control, a 3.2-m long straight section, and two bends with a midchannel radius of curvature of $1.49 \mathrm{~m}$; the sinuous section had a sinuosity of 1.2, defined as the ratio of along-stream length of the channel to the straight line distance. The channel model slope was $3 / 50$ radians ( $3.4^{\circ}$ or $6 \%$ gradient), and the channel terminated $0.1 \mathrm{~m}$ off the floor. A schematic (along with the coordinate system) and a photograph of the channel model are shown in Figures 2a and 2b.

Saline fluid (with temperature in the range of $21-22^{\circ} \mathrm{C}$ ) was pumped into the top of the channel, forming a gravity current that flowed along the channel and off the end. The basal $0.1 \mathrm{~m}$ of the 13-m diameter pool operated as a sump for the denser saline fluid to accumulate. This accumulated dense fluid was drained at a rate of $20 \mathrm{~m}^{3} \mathrm{hr}^{-1}\left(5.55 \mathrm{~L} \mathrm{~s}^{-1}\right)$ to limit in-channel ambient fluid modification. Two long metal rails were positioned to either side of the channel model across the full width of the flume to carry a computerized platform, which could be positioned at any point along the channel. The platform itself contained the controls for two Schneider slides oriented in traverse and vertical directions, enabling xyz control.

The pool was filled with fresh water to a depth of $1 \mathrm{~m}$, and experiments were performed after the ambient fluid was spun up to solid body rotation over a period of at least $12 \mathrm{hr}$. For the fastest rotation rates of $\Omega= \pm 0.167 \mathrm{rad} \mathrm{s}^{-1}$, the water surface would form a parabola with a height of $h_{p}=\left(\Omega^{2} / 2 \mathrm{~g}\right) R^{2}$. Hence, the difference in water elevation over the radius of the tank $(R=6.5 \mathrm{~m})$ is order $0.06 \mathrm{~m}$, i.e., much less than the $6 \%$ bottom slope of the channel. There were two measurement positions along the channel, namely, position X1 and position X2. Position X1 was located at the straight section and defined as $0.56 \mathrm{~m}$ upstream from the start of the straight channel (Figure 2a). Position X2 was placed at the second bend apex (which was a left-turning bend, as looking downstream) and located $7.01 \mathrm{~m}$ downstream of position X1 (Figure 2a). As noted in Table S2, a total of 22 experiments, comprising 11 Rossby conditions at two positions along the channel, were performed with rotation rates in the range of $-0.167 \leq \Omega \leq+0.167 \mathrm{rad} \mathrm{s}^{-1}$. Note that at the Equator, where the Coriolis force is absent $\left(f=0 \mathrm{rads}^{-1}\right)$ it is easier to plot $1 / R o_{W}$ or $1 / R o_{R}=0$ in comparison to $R o_{W}$ or $R o_{R}=\infty$. Thus, for convenience the inverse of the Rossby number is used in all plots in this study, as per prior work (Cossu et al., 2015). The density of the inflow was reduced to approximately $1,010 \mathrm{~kg}$ 
$\mathrm{m}^{-3}$ as a result of mixing in the inlet box. The data used in the analysis were collected after the saline current had flowed for $15 \mathrm{~min}$, as after this time the flow density had become constant at the measurement sections. Details of the flow parameters are documented in Table S3.

A Microscale Conductivity and Temperature Instrument (MSCTI) from Precision Measurement Engineering was used to determine the density structure of the gravity current. The density data correspond to a timeaveraged density that was averaged over 167 density data points that were collected over a period of 15 min. Details of its use are provided in the supplemental files.

A pair of Nortek Vectrino Acoustic Doppler Velocimeter Profiler (ADVP, Vectrino profiler) probes were used for mapping three-dimensional flow velocities at a frequency of $100 \mathrm{~Hz}$. Details of the use of the ADVP are provided in the supporting documents. The ADVP can measure three component velocities at 30 measurement points over a depth range of $0.03 \mathrm{~m}$, with this zone starting $0.04 \mathrm{~m}$ below the probe head. However, those measurement ranges are adjustable based on the situation of the experiment, which will be further explained in this section. Movement of ADVP probes on the programmable automatic traverse in the $y$ and $\mathrm{z}$ planes allowed detailed velocity profiles to be undertaken at spatial positions X1 and X2. In each position a total of 13 ADVP locations existed within the channel (as shown in Figure 2c), consisting of 11 locations at the centerline and two locations $0.15 \mathrm{~m}$ to the right and left of the centerline. Velocity profiles were sampled at each measurement location for $60 \mathrm{~s}$. Hence, each experimental measurement period lasted for approximately $15 \mathrm{~min}$, considering the elapsed time for movement between measurement locations. For each case, maximum time-averaged stream-wise velocities, $\langle U\rangle_{\max }$, were computed within the lowermost $30 \mathrm{~mm}$ of the current. Maximum time-averaged spanwise velocities, $\langle V\rangle_{\max }$, were computed within the lowermost $h_{U \max }$ of the current, where $h_{U \max }$ is the elevation of the maximum time-averaged stream-wise velocity. Error bars represent one standard error from the mean (68.2\% confidence intervals) and were estimated using the equations provided by Benedict and Gould (1996).

The pair of ADVPs in use were named ADVP1 and ADVP2 with the former being the basal probe (Figure 2d). ADVP1 was mounted at the center of the channel at a distance of $0.07 \mathrm{~m}$ from the bottom at position X1. However, for position X2 this distance ranged from 0.063 to $0.067 \mathrm{~m}$ depending on lateral position, suggesting that there was some small-scale lateral slope variation at the bend apex. ADVP1 measured three component velocities at 28 measurement points over a depth range of $0.028 \mathrm{~m}$, with this zone starting $0.042 \mathrm{~m}$ below the probe head. On the other hand, ADVP2 produced three component velocity profiles at 21 measurement points over a depth range of $0.021 \mathrm{~m}$, with this zone starting $0.045 \mathrm{~m}$ below the probe head. The offsets in the $\mathrm{x}$ and $\mathrm{z}$ directions for ADVP2 (relative to ADVP1) were 0.11 and $0.021 \mathrm{~m}$, respectively, suggesting the two ADVPs had a $0.009 \mathrm{~m}$ overlap in the profiles they produced. The details of the position of the ADVPs are illustrated in Figure 2d. Note that the $0.11 \mathrm{~m}$ distance between the two ADVPs in the $\mathrm{x}$ direction was required as this minimized side lobe interference but resulted in locating the two ADVPs at slightly different parts of the bend. This led to some discrepancy in consecutive downstream or cross-stream velocity profiles, as will be observed later in the results section. In most of the figures and calculations in this paper we solely focus on the data collected by ADVP1, which consists of the bottom $0.028 \mathrm{~m}$ of the gravity current.

Once the data were filtered and transformed and the bed was identified, the near-bed shear velocity $\left(U^{*}\right)$ was estimated from Reynolds stress components, allowing calculation of the dimensionless drag coefficient $C_{D}$ (Cossu \& Wells, 2012; Dallimore et al., 2001). The magnitude of the shear stress can be expressed as $\tau=\rho U^{* 2}$, which is the vector sum of the $\mathrm{x}$ and $\mathrm{y}$ components, i.e., $|\tau|=\left(\tau_{x x}{ }^{2}+\tau_{y y}{ }^{2}\right)^{1 / 2}$. Following Gray et al. (2006), these shear stresses near the base of a gravity current can be defined in terms of Reynolds stress, i.e., $\tau_{x x}=\rho\left\langle u^{\prime} w^{\prime}\right\rangle$, where $u^{\prime}$ and $w^{\prime}$ are downstream and vertical velocity fluctuations, respectively and angle brackets again denote a time-average. In the region below the velocity maximum of depth $h$, the near-bed shear velocity $\left(U^{*}\right)$ was calculated from the vector sum of downstream and cross-stream Reynolds stresses as

$$
U^{*}=\left(<u^{\prime} w^{\prime}>^{2}+<v^{\prime} w^{\prime}>^{2}\right)^{1 / 4}
$$

where $u^{\prime}, v^{\prime}$, and $w^{\prime}$ are downstream, cross-stream, and vertical velocity fluctuations, respectively, and $\left\langle u^{\prime} w^{\prime}\right\rangle$ and $\left\langle v^{\prime} w^{\prime}\right\rangle$ are the Reynolds stress components along and perpendicular to the gravity current. In this paper 
we will report near-bed means of $U^{*}$ over the depth $h$. The drag coefficient $C_{D}$ was then calculated in terms of the near-bed velocity maximum $U_{\max }$ as

$$
C_{D}=\frac{U^{* 2}}{U_{\max }^{2}} .
$$

The near-bed maximum velocity $U_{\max }$ is used in this expression for pragmatic purposes, as the ADVP measurements of $U^{*}$ are only in the bottom $0.028 \mathrm{~m}$.

\section{Results}

\subsection{Overview of Experiments}

The set of 22 experiments performed on the large-scale rotating facility in Grenoble provides the highest Reynolds numbers to date for gravity currents that were influenced by rotation, with values in the range of $R e \sim 14,000-27,000$ (ignoring the flows which were so heavily tilted that centerline values are unreliable; see later discussion). In particular, the flows were at least five times deeper, six times wider, and velocities were measured at 10 times the vertical resolution of previous work by Cossu and Wells (2010) or Cossu et al. (2010), allowing measurements within the boundary layers to now be made. This allows us to gain insight into how turbulence structures and velocities are modified in conditions resembling high and low latitudes. Analysis of different aspects of the flow such as downstream and cross-stream velocities, density fluctuation, shear velocity, drag coefficient, and Ekman number is here displayed through various images and figures in this section. Table S2 details our calculated dimensionless numbers (Rossby, Reynolds, Froude and Ekman numbers), the drag coefficient and Ekman depth corresponding to each experiment, and the different variables by which those numbers were determined.

The evolution of a gravity current flowing through a channel can change dramatically under the influence of the Coriolis force. The dynamics of such a flow going around a bend is visually displayed in Figure 3 through two groups of photos with and without rotation, which were taken from the top of the experimental channel. Comparing the set of photos in Figures $3 \mathrm{a}$ and $3 \mathrm{~b}$, it can immediately be seen that the Coriolis force can have a strong influence on the dynamics of the current. As can be seen in Figure $3 \mathrm{a}$, corresponding to $R o_{R}=\infty$ (flow with no rotation), the current fills the full width of the channel as it moves along the curved channel. On the other hand, in Figure $3 \mathrm{~b}$, corresponding to $R o_{R}=-0.15$, strong Coriolis force deflects the gravity current towards the left bank of the channel. One surprising result is that, when comparing the two groups of images, the head of the flow moved faster around the bend under the effect of Coriolis force, opposite to the expectation for geostrophic flows in straight channels, where Coriolis force tends to decrease the downstream velocity (Cossu \& Wells, 2010). The quantitative shifts in velocity will be further documented and analyzed with the incorporation of the ADVP measurements later in this section.

The lateral shift of the density and velocity core due to the Coriolis force means that some of the experimental parameters in Table S2 have to be interpreted carefully. In all of the 22 experimental cases, profiles of velocity and density were collected at the centerline at positions X1 and X2. However, in a few cases values at the centerline location are not representative of the real conditions within the current. For example, all the flows are supercritical in the straight section (position X1), with the exception of experiment 5X1S corresponding to $R o_{W}=-0.55$ and $\mathrm{Fr}=0.87$. Such a small Froude number reflects the very low recorded velocity in this case, where the effect of high rotation caused a steep lateral interface angle deflecting the density and velocity core to the left-hand side of the channel rather than the centerline. Similarly, the small Reynolds numbers in the apex section (position X2) corresponding to experiment 5X2S (Re $=6418, R o_{R}=-0.15$ ) and experiment $5 \mathrm{X} 2 \mathrm{~N}\left(R e=6189, R o_{R}=+0.06\right)$ are a consequence of the gravity current being strongly deflected to the inner (as illustrated in Figure $3 \mathrm{~b}$ ) and outer bends, respectively, and hence, the lower velocity measured reflects the edge of the gravity current rather than that of the faster moving core. For this reason, velocity data from these three experiments have not been used during figure production. The proceeding discussion focuses on velocity and density profiles collected during experiments 3X1S, 6X1EQ, and $3 \mathrm{X} 1 \mathrm{~N}$ at position $\mathrm{X} 1\left(R o_{W}=-2.76, \infty\right.$, and +2.83$)$ and experiments $3 \mathrm{X} 2 \mathrm{~S}, 6 \mathrm{X} 2 \mathrm{EQ}$, and $3 \mathrm{X} 2 \mathrm{~N}$ at position $\mathrm{X} 2\left(R o_{R}=-0.81, \infty\right.$, and +0.8$)$. After plotting several figures for different experimental cases, and extensive 

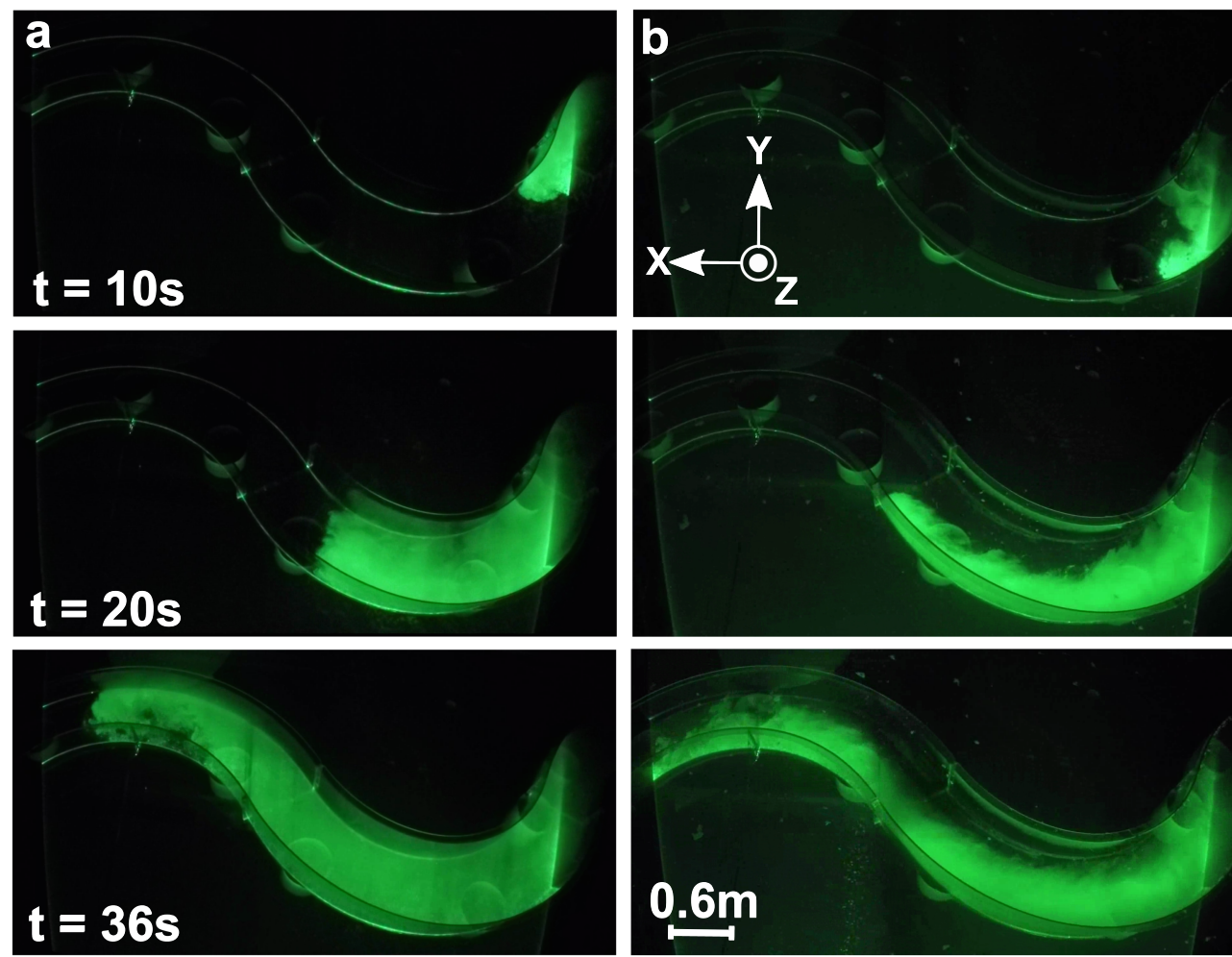

Figure 3. A set of photos showing flow with (a) no rotation $\left(R o_{R}=\infty\right.$, experiment $\left.6 \mathrm{X} 2 \mathrm{EQ}\right)$, where the gravity current alternates between banks and (b) strong rotation $\left(R o_{R}=-0.15\right.$, experiment $\left.5 \mathrm{X} 2 \mathrm{~S}\right)$, where the flow is always confined on the left bank and very little of the gravity current would pass through the central measurement location. Note that at $36 \mathrm{~s}$ the front of the current in the case of $R o_{R}=-0.15$ has progressed further around the left-turning bend compared to the nonrotating case, indicating that in the rotating case the maximum speed of the current is greater than the nonrotating case. The gravity current was visualized using laser induced fluorescence. Movies S1 (corresponding to $R o_{R}=\infty$ ) and Movie S2 (corresponding to $R o_{R}=-0.15$ ) are included in the supporting information.

comparisons, we concluded that the above mentioned cases provide the best insight of the effect of the Coriolis and centrifugal forces on the current.

\subsection{Velocity and Density Profiles}

Time-averaged downstream and cross-stream velocities and density profiles at the centerline are displayed in Figures $4 \mathrm{a}-4 \mathrm{c}$ for the straight section (representing $R o_{W}=-2.76, \infty,+2.83$ ) and Figures $4 \mathrm{~d}-4 \mathrm{f}$ for the bend apex (representing $R o_{R}=-0.81, \infty,+0.8$ ). The depth of the flow increases from approximately 0.15 $\mathrm{m}$ at the straight section to $0.22 \mathrm{~m}$ at the second bend apex. Coriolis force has little influence on the downstream velocity profiles in the straight section (Figure 4a), whereas changes are observed in the bend apex as a function of that force (Figure $4 \mathrm{~d})$. For the cases of no rotation $\left(R o_{R}=\infty\right)$, and a positive rotation $\left(R o_{R}=\right.$ +0.8 ), where the centrifugal and Coriolis forces are in the same direction in the bend, the downstream velocity profiles show a maximum close to the bed, a rapid initial decline above the maximum, and then a zone up to $\sim 0.1 \mathrm{~m}$, where velocity declines relatively slowly (Figure $4 \mathrm{~d}$ ). In the case of $R o_{R}=+0.8$ there is a very limited decline in downstream velocity in this basal $0.1 \mathrm{~m}$ zone, with the flow exhibiting a second local velocity maximum at $\sim 0.1 \mathrm{~m}$ (Figure $4 \mathrm{~d}$ ). In contrast, for the case where the Coriolis force opposes the centrifugal force $\left(R o_{R}=-0.81\right)$, there is a continuous and rapid decline in flow velocity above the velocity maximum, which again is located close to the bed (Figure 4d). Consequently, at a height of $0.1 \mathrm{~m}$ the downstream velocities in the case for $R o_{R}=-0.81\left(U_{R o}=-0.81=0.10 \mathrm{~m} \mathrm{~s}^{-1}\right)$ are $0.05 \mathrm{~m} \mathrm{~s}^{-1}$ lower than for the $R o_{R}=+0.8$ case $\left(U_{R o}=+0.8=0.15 \mathrm{~m} \mathrm{~s}^{-1}\right)$ and $0.06 \mathrm{~m} \mathrm{~s}^{-1}$ lower than for $R o_{R}=\infty\left(U_{R o=\infty}=0.16 \mathrm{~m} \mathrm{~s}^{-1}\right)$.

The cross-stream velocity profiles also show some interesting trends. In a general comparison between Figures $4 \mathrm{~b}$ and $4 \mathrm{e}$, smaller time-averaged cross-stream velocity values $(\langle V\rangle)$ are observed in the straight section, confirming the effect of the centrifugal force in the bend apex. For the straight channel, the blue cross-stream profile for $R o_{\mathrm{W}}=+2.83$ in Figure $4 \mathrm{~b}$ shows a secondary circulation of $\langle V\rangle=-0.01 \mathrm{~m} \mathrm{~s}^{-1}$ to the left-hand side, very close to the base. While there is some overlap of error bars in this turbulent 

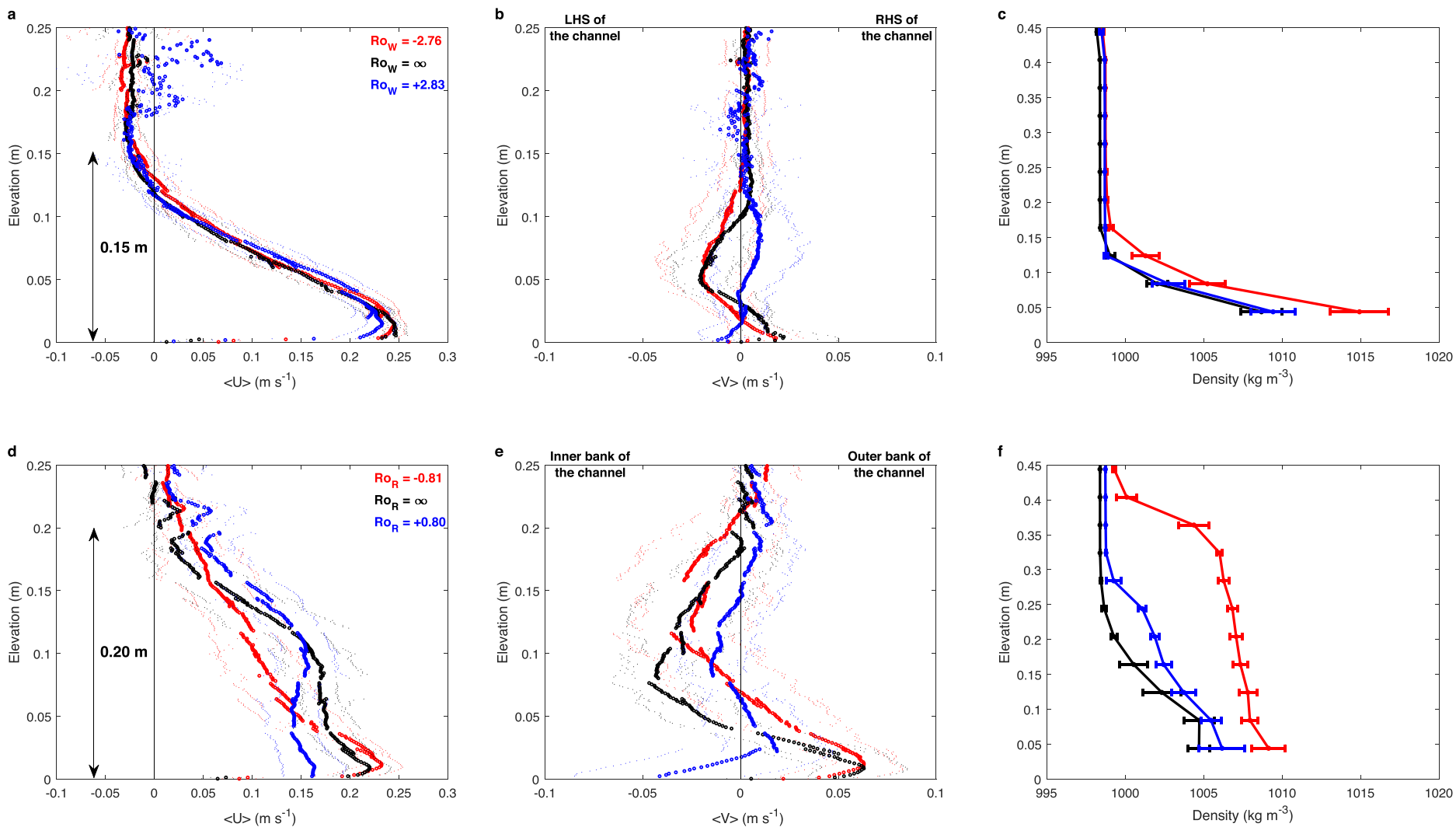

Figure 4. Time-averaged downstream $\langle U\rangle$, cross-stream $\langle V\rangle$ velocity (from ADVP1 and ADVP2), and density profiles (from MSTCI conductivity probe), at the centerline in (a), (b), and (c) the straight section corresponding to experiments $3 \mathrm{X} 1 \mathrm{~S}, 6 \mathrm{X} 1 \mathrm{EQ}$, and $3 \mathrm{X} 1 \mathrm{~N}\left(R o_{W}=-2.76, \infty,+2.83\right)$ and $(\mathrm{d}),(\mathrm{e})$, and (f) the bend apex section corresponding to experiments $3 \mathrm{X} 2 \mathrm{~S}, 6 \mathrm{X} 2 \mathrm{EQ}$, and $3 \mathrm{X} 2 \mathrm{~N}\left(R o_{R}=0.81, \infty,+0.8\right)$. The basal point for density measurements started $4.4 \mathrm{~cm}$ above the base. Note that the two lines for each Rossby number plotted in (e), and the breaks between the velocity data in other plots, reflect small variations in the downstream position of the two ADVPs (see text for details). Error bars represent one standard error from the mean (68.2\% confidence intervals) and were estimated using the equations provided by Benedict and Gould (1996).

region, the mean velocity for $R o_{\mathrm{W}}=-2.83$ is significantly different to that of $R o_{\mathrm{W}}=-2.83$. The across stream velocity, below the depth of the downstream velocity maxima of the gravity current is deflected to the righthand side (by velocities as high as $\langle V\rangle=0.011 \mathrm{~m} \mathrm{~s}^{-1}$ ), followed by a small return flow to the left at the surface of the gravity current $(0.15 \mathrm{~m}$ above base). This circulation is consistent with the sense of flow in straight channels, which also have the Coriolis force in the Northern Hemisphere sense. An almost mirrored velocity profile is observed for $R o_{W}=-2.76$, simulating the Southern Hemisphere. The positive rotation in the Northern Hemisphere locates the across stream velocity, at and just above the depth of the downstream velocity maxima of the current, towards the right-hand side of the channel and is accompanied by return flows to the left at the lower, and possibly upper, parts of the density current. In the Southern Hemisphere, the negative rotation sends the across stream velocity, at the depth of the downstream velocity maxima of the current, to the left with the two return flows towards the right-hand side of the channel. This observation is in agreement with the flow fields reported by Johnson and Ohlsen (1994), Davies et al. (2006), and Cossu et al. (2010). Note that the deflection of the flow for $R o_{W}=\infty$ in the straight section in Figure $4 \mathrm{~b}$ is likely due to a pair of cells converging at the surface and diverging at the bottom, similar to the secondary flow observed in straight rivers (Nezu et al., 1993). In the absence of rotation at the second bend apex $\left(R o_{R}=\infty\right)$, the secondary flow close to the bottom is towards the outer bend accompanied by a return flow near the surface (Figure $4 \mathrm{e}$, the black profile). Similar behavior has been reported in nonrotating submarine channel experiments (Corney et al., 2006, 2008; Cossu \& Wells, 2010; Dorrell et al., 2018; Keevil et al., 2006).

In a left-turning bend for the Northern Hemisphere, such as the case of $R o_{R}=+0.8$, the centrifugal and Coriolis forces are acting in the same direction so that the bulk of the current is deflected towards the 
outer bank of the channel (Figure 4e, the blue profile). However, in a sharp contrast to the $R o_{R}=\infty$ case, where the basal flow is in the direction of the centrifugal force, the basal flow for the $R o_{R}=+0.8$ is reversed and in the opposite direction to the centrifugal force towards the inner bank of the channel (Figure 4e). In the Southern Hemisphere case $\left(R o_{R}=-0.81\right)$, where the centrifugal and Coriolis forces are opposing, the base of the gravity current is towards the outer bend, with a return flow above this (the red profile in Figure 4e). These observations of the direction of middepth flows are in broad agreement with the observations of the direction of cross-stream flow in Figure 3 of Cossu and Wells (2010). We note that this previous study lacked detailed measurements of the basal flows which makes it hard to compare details on basal flows, especially as the Ekman boundary layer was very likely below the lowest measurement in Cossu and Wells (2010). It is also important to note that while the cross-stream velocity at the depth of the velocity maxima is directed to the RHS of the channel for all three cases, the cross-stream velocity for $R o_{R}=+0.81$ (blue curve) at this depth is lower than either the $R o_{R}=-0.8$ or $R o_{R}=\infty$ case. It is likely that this lower cross-stream velocity to the RHS is due to the downstream velocity being lower for $R o_{R}=+0.81$ (blue curve in Figure 4d) and due to the increased vertical shear, as the near-bed flows now go to LHS, rather than the RHS for the two cases of $R o_{R}=-0.8$ or $R o_{R}=\infty$. Note that the basal flows will be discussed further below.

The time-averaged density profiles in Figures $4 \mathrm{c}$ and $4 \mathrm{f}$ have a similar trend to the downstream velocity profiles (Figures 4a and 4d). Both figures indicate that the depth of the flow has increased at the apex. Furthermore, there are significant density differences along the channel, as the maximum density of about $1,015 \mathrm{~kg} \mathrm{~m}^{-3}$ in the straight section (Figure 4c) decreases to $1,009 \mathrm{~kg} \mathrm{~m}^{-3}$ in the apex (Figure 4f). The increase in the depth of the currents (by 25\%), along with the decreases in density (by 6\%) and velocity (by 14\%), indicate that the flow has become deeper, slower, and more dilute with downstream distance, and thus, entrainment of light fluid at the upper interface has occurred. It is worth noting that the density structure for $R o_{w}=-0.81$ in Figure $4 \mathrm{f}$ is different to other two cases, suggesting that the internal density layers are being banked up against outer bend. Finally, the downstream velocity and density profiles in the straight section follow a very similar pattern for the three Rossby numbers (Figures 4a and 4c). However, this similar trend is not observed at the second bend apex as a result of the occurrence of the centrifugal force there (Figures $4 \mathrm{~d}$ and $4 \mathrm{f}$ ).

\subsection{Velocity Dynamics in the Bottom Boundary Layer}

The dynamics of sediment erosion and deposition occur within the bottom boundary layer of a gravity current, and we now focus on this region of the flow, which in a supercritical flow also contains the velocity maximum. The maximum downstream velocity in a gravity current often occurs close to the bed, since stable stratification implies that the density-difference driving force is concentrated closer to the base of the flow, whereas mixing at the top interface generates drag into the flow (Meiburg \& Kneller, 2010; Turner, 1973). In Figure 5, the time-averaged downstream velocity profiles of the bottom $0.028 \mathrm{~m}$ are presented at three lateral locations for positions X1 and X2, under various Rossby numbers. The maximum time-averaged downstream velocity is very close to the bottom of the channel for all discussed Rossby numbers in both sections, with the distance being in the range of $h=0.006-0.026 \mathrm{~m}$. For the nonrotating case $\left(R o_{R}\right.$ or $\left.R o_{W}=\infty\right)$ in both positions $\mathrm{X} 1$ and $\mathrm{X} 2$ at the centerline location, the maximum downstream velocity decreases slightly from $0.25 \mathrm{~m} \mathrm{~s}^{-1}$ (occurring at $h=0.006 \mathrm{~m}$ ) at position X1 to $0.22 \mathrm{~m} \mathrm{~s}^{-1}$ (occurring at $h=0.008 \mathrm{~m}$ ) at position $\mathrm{X} 2$ (black profiles in Figures $5 \mathrm{~b}$ and $5 \mathrm{e}$ ). This reflects the downstream deceleration, thickening of the flow and the presence of centrifugally derived secondary flow at the second bend apex. The profile for the nonrotating case in all three y locations of the straight section (black profiles in Figures 5a-5c) would be expected to be roughly similar, and the small changes are likely related to possible inflow conditions or slight crossstream slopes in the channel model. Due to the effect of the Coriolis force and secondary circulation, the maximum downstream velocity in the straight section at the centerline (Figure $5 \mathrm{~b}$ ) is greatest for the nonrotating and the positive rotation cases $\left(\langle U\rangle_{\max }=0.25 \mathrm{~m} \mathrm{~s}^{-1}\right)$, with some slight decreases for both faster negative rotation rates. This slight slowing of the flow with increased Coriolis force is consistent with the scaling of the "Nof velocity" (Ivanov et al., 2004); however, in the present experiments, it may also reflect the lateral tilting of the flow and thus, measurement of a slower part of the flow at the centerline. We note that the Nof velocity is a scaling for a depth-averaged flow and does not provide a theory for the internal velocity structure of a gravity current. 


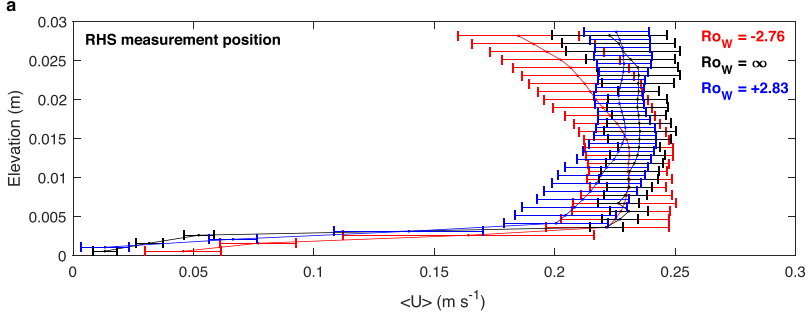

b

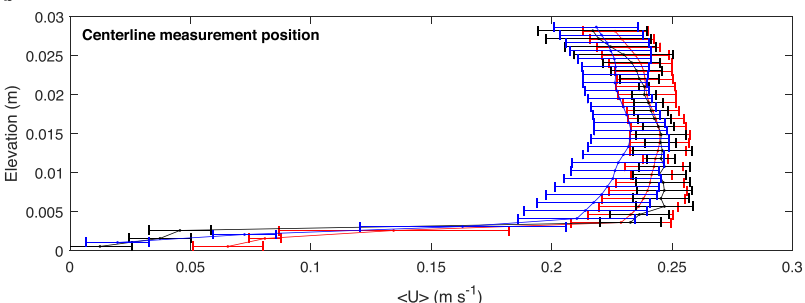

c

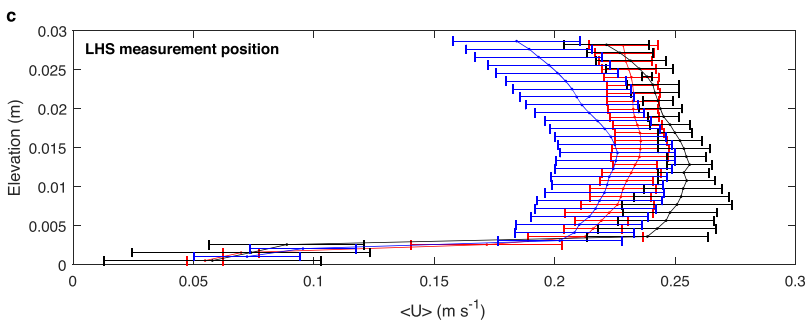

d
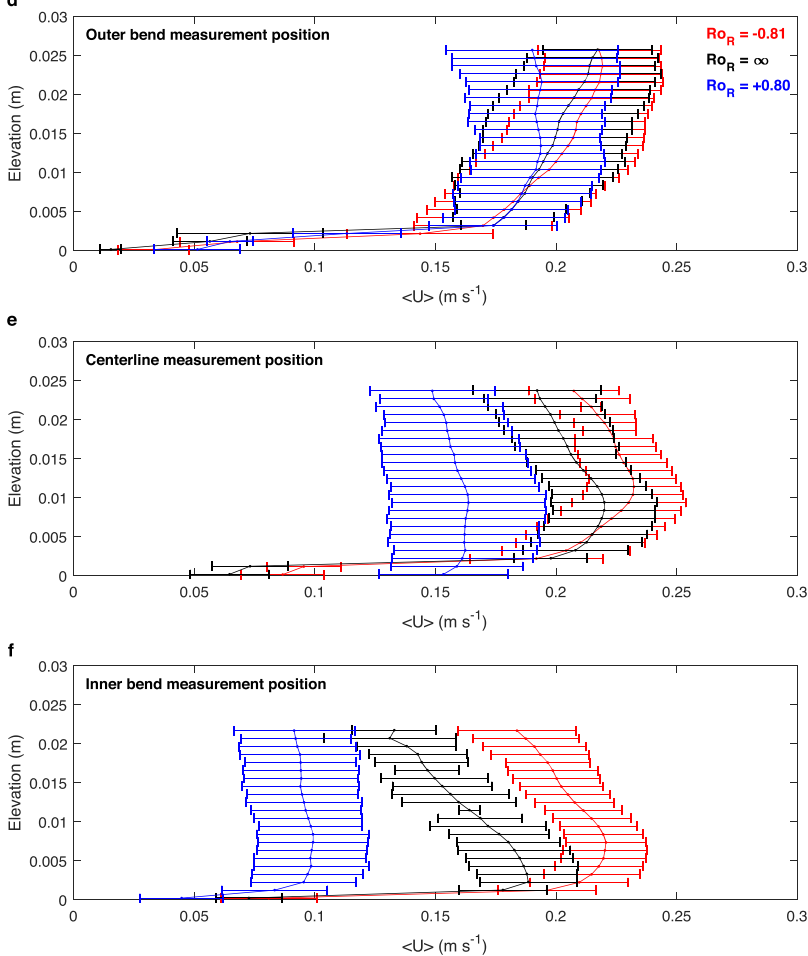

Figure 5. Time-averaged downstream velocity profiles of the bottom $0.028 \mathrm{~m}$ (from ADVP1) at three measurement locations across the channel for (a), (b), (c) the straight section $\left(R o_{W}=-2.76, \infty,+2.83\right.$ corresponding to experiments $3 \mathrm{X} 1 \mathrm{~S}, 6 \mathrm{X} 1 \mathrm{EQ}$, and $\left.3 \mathrm{X} 1 \mathrm{~N}\right)$ and $(\mathrm{d}),(\mathrm{e}),(\mathrm{f})$ the bend apex section $\left(R o_{R}=-0.81, \infty,+0.8\right.$ corresponding to experiments $3 \mathrm{X} 2 \mathrm{~S}, 6 \mathrm{X} 2 \mathrm{EQ}$, and $3 \mathrm{X} 2 \mathrm{~N})$.Error bars represent one standard error from the mean (68.2\% confidence intervals) and were estimated using the equations provided by Benedict and Gould (1996).

In the bend apex, faster downstream velocities are observed in the basal zone for the negative Rossby number case $\left(R o_{R}=-0.81\right)$, in comparison to those for positive Rossby numbers $\left(R o_{R}=+0.8\right)$ and the case with no rotation $\left(R o_{R}=\infty\right)$ (Figures $\left.5 \mathrm{~d}-5 \mathrm{f}\right)$. Note that in Figure $5 \mathrm{~d}$, while the maximum downstream velocity $\left.(<U\rangle_{\max }\right)$ is always within the range of $h=0.006-0.026 \mathrm{~m}$ of the bed, for $R o_{R}=\infty$ and -0.81 the maximum occurs at a higher flow depth compared to the other cases. However, as described earlier, there are contrasting changes in downstream velocities within the bulk of the flow, as identified in the centerline profiles (Figure 4d), with flow decelerating with height far more rapidly in the negative Rossby case than in the other two cases. The faster basal velocities observed for the negative Rossby case may provide an explanation for the observation of faster head velocities observed from planform photographs in Figure $3 \mathrm{~b}$ for a negative Rossby case $\left(R o_{R}=-0.15\right)$. This is interesting as it is opposite to the usual statement that the Coriolis force tends to slow the current down, as expressed by the Nof velocity, where $U_{\text {geo }} \sim g^{\prime} S / f$, in which $S$ is slope and $g^{\prime}$ the reduced gravity (Ivanov et al., 2004). However, this Nof velocity represents the bulk flow, rather than the variations in velocities with height observed herein. While there may be trends in the data as described above, the large magnitude of the error bars casts some doubt on their veracity. We subsequently refine the analysis to be able to quantify trends between runs.

There is symmetry in the sense of the secondary flow between positive and negative rotation rates (Northern and Southern Hemispheres) in the straight channel (Figures 6a-6c). These figures show the time-averaged cross-stream velocity profiles of the bottom $0.028 \mathrm{~m}$ of the gravity current. There is a basal flow directed to the right-hand side of the channel for $R o_{W}=-2.76$ and to the left-hand side for $R o_{W}=+2.83$. The reasons for the deflections in the nonrotating case $\left(R o_{W}=\infty\right)$ in the straight section were discussed earlier and are likely due to paired secondary cells and/or to small lateral variations in inflow conditions.

The near-bed cross-stream velocities are much larger (in some cases up to 10 times) in the channel bend than in the straight section, despite the overall decrease in downstream velocity with distance (Figures 6d-6f). Furthermore, the direction of cross-stream flows varies with distance across the channel, in contrast to the symmetry between positive and negative Rossby numbers as observed in the straight section 


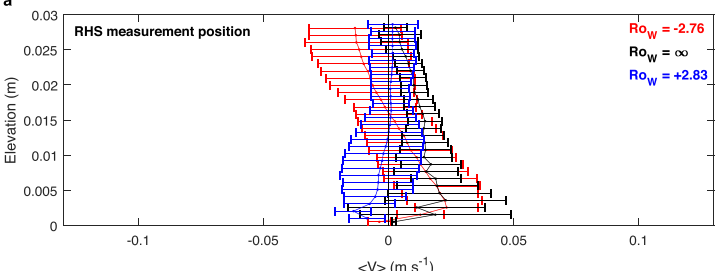

b
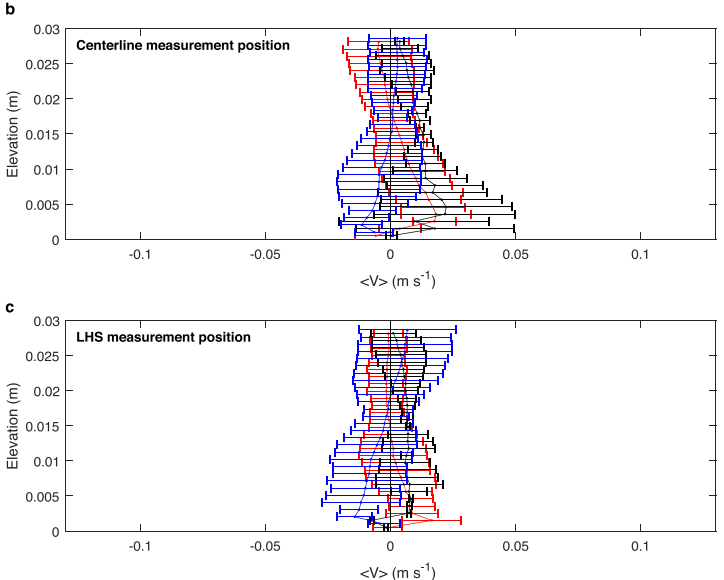

d
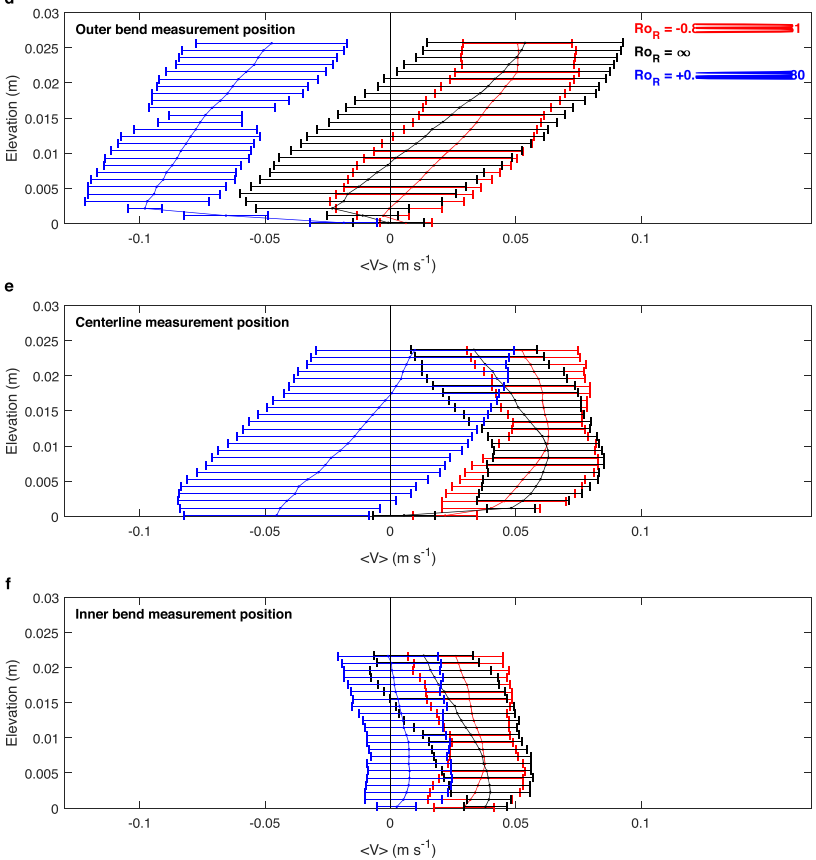

Figure 6. Time-averaged cross-stream velocity profiles of the bottom $0.028 \mathrm{~m}$ (from ADVP1) at three measurement locations across the channel for (a), (b), (c) the straight section $\left(R o_{W}=-2.76, \infty,+2.83\right.$, corresponding to experiments $3 \mathrm{X} 1 \mathrm{~S}, 6 \mathrm{X} 1 \mathrm{EQ}$, and $\left.3 \mathrm{X} 1 \mathrm{~N}\right)$ and $(\mathrm{d}),(\mathrm{e}),(\mathrm{f})$ the bend apex section $\left(R o_{R}=-0.81, \infty,+0.8\right.$, corresponding to experiments $3 \mathrm{X} 2 \mathrm{~S}, 6 \mathrm{X} 2 \mathrm{EQ}$, and $3 \mathrm{X} 2 \mathrm{~N}$ ). In (a)-(c) a positive $V$ is a flow to the right-hand side of the channel. In (d)-(f) a positive $V$ is a flow towards the outer bank of the channel. The position indicated on each figure corresponds to the three measurement locations within the channel along the $y$ axis. Error bars represent one standard error from the mean (68.2\% confidence intervals) and were estimated using the equations provided by Benedict and Gould (1996),

(Figures $6 \mathrm{~d}-6 \mathrm{f})$. For the nonrotating case $\left(R o_{R}=\infty\right)$, basal flow at the centerline and inner bend positions is towards the outer bank of the channel (black profiles in Figures 6e and 6f) as might be expected with the centrifugal force dominating at the depth of the downstream velocity maximum (Corney et al., 2006, 2008; Dorrell et al., 2013). However, in the outer bend position there is a small area of flow towards the inner bend of the channel, up to a depth of $0.008 \mathrm{~m}$ (black profile in Figure $6 \mathrm{~d}$ ), perhaps reflecting a developing corner cell. Such corner cells are well known to form in rectangular channels, as a result of transverse gradients of Reynolds shear stress (Prandtl, 1925; Gessner, 1973; Yang et al., 2012). There is a very similar pattern for $R o_{R}=-0.8$, where the Coriolis and centrifugal forces work in opposition (red colored profile in Figures $6 \mathrm{~d}-6 \mathrm{f}$ ). However, for $\mathrm{Ro}_{R}=+0.8$, when the Coriolis and centrifugal forces work in the same direction, secondary flow near the bed is directed strongly towards the inner bend of the channel at the outer bend and centerline positions, thus in the opposite direction to the centrifugal force (blue profile in Figures $6 \mathrm{~d}$ and $6 \mathrm{e}$ ). This change in direction of near-bed flow is an important result that will be discussed later in terms of potential sediment transport.

When considering the differences in velocity structure between the spanwise positions, it should also be remembered that the bulk of the gravity current is also being laterally deflected (Figure 4). Based on previous descriptions of upper interface slope in Wells and Cossu (2013), for $R o_{R}=\infty$ the gravity current would be located more towards the outer bend, with an upper interface that slopes down towards the inner bend. For $R o_{R}=-0.8$, we expect that the upper interface is nearly flat. However, for $R o_{R}=+0.8$ where the Coriolis force is in same direction as the centrifugal force, the upper interface will be super elevated towards the outer bend of the channel, as the gravity current is strongly deflected in that direction. This would leave very little of the gravity current near the inner bend measurement position and may explain the low velocities at that location (blue colored profile in Figure 6f). However, since these low velocities do not mirror the direction of near-bed flow in the other lateral positions, this provides further evidence that a corner cell is developing (Cossu \& Wells, 2010).

The key result of Figures $6 \mathrm{~d}$ and $6 \mathrm{e}$ is the reversal of the basal flow in the channel bend when the Coriolis force is sufficiently high to overcome the centrifugal force. The basal flow that is oriented in the opposite 
direction to the centrifugal force, in the case of $R o_{R}=+0.8$, likely reflects what is usually described as an "Ekman boundary layer" (i.e., Darelius, 2008; Johnson \& Sanford, 1992), but a stratification-modified Ekman boundary layer might be more accurate for cases such as the present, where the Coriolis force dominates underneath a strongly stratified region. The bottom boundary layer of a gravity current affected by the Coriolis force has been observed to exhibit a secondary circulation that opposes the circulation higher in the water column in field studies (Johnson \& Sanford, 1992; Umlauf \& Arneborg, 2009a, 2009b) and in straight channel experiments (Darelius, 2008; Davies et al., 2006; Johnson \& Ohlsen, 1994), but this is the first time that this has been recorded experimentally in sinuous subaqueous channels.

There are strong changes to the maximum near-bed (i.e., $<0.028 \mathrm{~m}$ ) values of downstream and cross-stream velocities as the Rossby number varies (Figure 7). The dashed blue curves fitted to Figures $7 \mathrm{a}-7 \mathrm{c}$ were obtained using Matlab's fitnlm nonlinear regression model function using the inverse square standard errors as weights and eliminating outliers greater than 1.5 standard deviations from the model. It is worth noting that the current may not be detected by some off-center measurements because of the large surface elevation, especially at very low Rossby numbers in Figures 7a and $7 \mathrm{~b}$. The downstream velocity data in the straight section (Figure 7a) show a symmetric pattern around $1 / R o_{W}=0\left(R o_{W}=\infty\right)$, while there is a linear relationship between $1 / R o_{R}$ and $\langle U\rangle_{\max }$ for the bend apex case (Figure $7 \mathrm{c}$ ). For the straight channel case, downstream velocities decrease with higher rotation rates (higher inverse Rossby numbers, and thus lower Rossby numbers), as previously described by Cossu et al. (2010). In the apex section of our left-turning bend on the other hand, the density current has the fastest maximum downstream velocity when the Coriolis and centrifugal forces oppose each other $\left(1 / R o_{R}<0\right)$ and the slowest maximum downstream velocity when they act together $\left(1 / R o_{R}>0\right)$ (Figure $\left.7 \mathrm{c}\right)$. Figure $7 \mathrm{c}$ also shows that the maximum downstream velocity at the inner bank for $1 / R o_{R}=-6.66\left(R o_{R}=-0.15\right)$ has increased by $0.076 \mathrm{~m} \mathrm{~s}^{-1}(41 \%)$ compared to the no rotation case $\left(R o_{R}=\infty\right)$. This is in agreement with the head velocities observed in planform videos (Figure 3 ). Note that the small values of downstream velocity at the outer wall $\left(\langle U\rangle_{\max }=-0.01 \mathrm{~m} \mathrm{~s}^{-1}\right)$ for $1 / R o_{R}=-6.66$ $\left(R o_{R}=-0.15\right.$, the blue data point $)$ and the inner wall $\left(<U>_{\max }=-0.01 \mathrm{~m} \mathrm{~s}^{-1}\right)$ for $1 / R o_{R}=16.66\left(R o_{R}=\right.$ +0.06 , the green data point) in Figure 7c correspond to the absence of the gravity current at one side of the channel, where it was strongly deflected to the opposite side. The secondary circulation shifts towards the left- and right-hand sides of the straight channel (Figure $7 \mathrm{~b}$ ) and the inner and outer banks of the curved channel (Figure 7d), as the Rossby number changes. The straight channel section displays a largely symmetric distribution of the direction of cross-stream velocities (Figure 7b), discounting the fastest rotating experiments, where the velocity core was pushed to one extreme side or the other. The cross-stream velocities in the apex section in Figure 7d show a similar pattern to Figure 7b with a change in direction with the change in sign of Rossby number; however, there are two important differences. First, the magnitude of secondary flows is roughly twice as high in the apex, compared to the straight section. Second, it appears that there is a minimum positive inverse Rossby number of approximately $1 / R o_{R}=+1.25\left(R o_{R}=+0.8\right)$ needed before the near-bed flows change direction, suggesting that the Coriolis force needs to reach a certain threshold compared to the centrifugal force. For cases with Rossby numbers smaller than $R o_{R}=+0.8$, a stratification-modified Ekman boundary layer is present at the base of the flow that is oriented in the opposite sense to the centrifugal force.

\subsection{Lateral Density Differences in the Bottom Boundary Layer}

The changes in Coriolis force have a substantial effect on the lateral slope of the density interfaces within the gravity current as it moves in the channel (Figure 8). Previous work of Cossu and Wells (2010) and Wells and Cossu (2013) could only describe the upper interface of the density current, whereas in the present study we are able to examine the internal structure from the conductivity measurements made by the MSTCI conductivity probe. Figure 8 depicts the lateral differences of the near-bed density $(0.044 \mathrm{~m}$ above the bottom of the channel) as a function of rotation (inverse Rossby number) in the straight and apex sections. In the straight section, the dense fluid at the base of the current is deflected to the right- and the left-hand side at faster positive and negative rotation rates, respectively (Figure 8a). In contrast, at the bend apex in almost all cases the maximum density is deflected towards the outer bank, suggesting that the centrifugal force dominates this region of the flow (Figure 8b). Comparing Figures $8 \mathrm{a}$ and $8 \mathrm{~b}$ shows that density differences across the channel for the straight section vary between $-8.77<<\rho><7.75 \mathrm{~kg} \mathrm{~m}^{-3}$, whereas at the bend apex almost all of the lateral density anomalies are in the range $0<<\rho><7.89 \mathrm{~kg} \mathrm{~m}^{-3}$. In order to understand these numbers, it is 

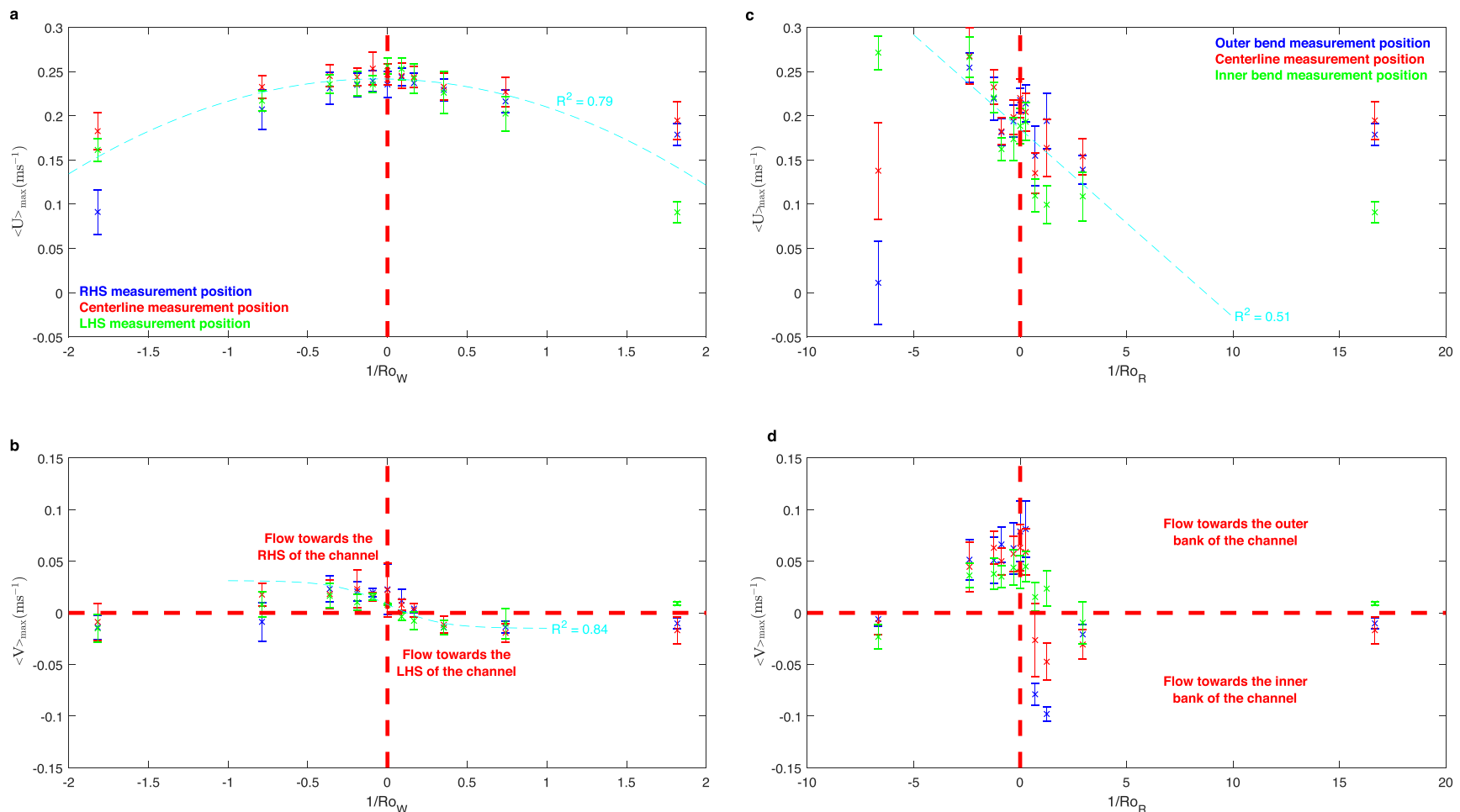

Figure 7. Time-averaged maximum downstream $\left(\langle U\rangle_{\max }\right)$ and cross-stream $\left(\langle V\rangle_{\max }\right)$ velocities at the three measurement locations across the channel as a function of rotation for (a), (b) the straight and (c), (d) bend apex sections. The blue dashed lines emphasize the general trends that are apparent in the data, along with $R^{2}$ values. Comparing (a) and (c), the downstream velocity data in the straight section depicts a symmetric pattern around $1 / R o_{W}=0$, whereas in the bend section there is an asymmetric pattern. In both (b) and (d) the cross-stream velocity data has a symmetric distribution in terms of the direction ignoring the low Rossby numbers. Comparing (b) and (d), the magnitude of $\langle V\rangle_{\max }$ in the apex in almost two times larger than the straight section. Furthermore, in (d) the near-bed flow changes direction at $1 / R o_{R}=+1.25\left(R o_{R}=+0.8\right)$.

important to remember that the maximum density for $R o_{W}=\infty$ in Figure $4 \mathrm{c}$ is $1,015 \mathrm{~kg} \mathrm{~m}^{-3}$, but at the bend apex downstream dilution means that this maximum density is $1,009 \mathrm{~kg} \mathrm{~m}^{-3}$ for $R o_{R}=\infty$ in Figure $4 \mathrm{f}$. The large density anomaly values in Figure 8a represent the maximum possible vertical density difference in Figure $4 \mathrm{c}$, implying that all of the density current is at one side of the channel, and fresh water is at the other. The smaller density anomalies in Figure $8 \mathrm{~b}$ represent the maximum density anomaly of the diluted density current at the bend apex and are in line with the maximum vertical density difference in Figure $4 \mathrm{f}$. It is significant that almost all values in Figure $8 \mathrm{~b}$ are positive (except for $1 / R o_{R}=6.66 ; R o_{R}=-0.15$ ) meaning that the flow is always denser at the outer bank and implies that the centrifugal force is dominant in always moving this dense core in the same direction. In addition, the density differences at the outer bank (blue points in Figure 8b) are generally higher than the density differences at the inner bank (green points in Figure 8b), therefore showing increasing density contrast towards the outer bank.

\subsection{Shear Velocities and Drag Coefficients}

The shear velocity $\left(U^{*}\right)$ calculated from equation (8) is slightly larger on average in the bend apex than the straight section. The mean values of the shear velocity calculated over three measurement locations across the channel are plotted for both positions X1 and X2 as a function of rotation (inverse Rossby number) in Figure 9. In most of the experiments, this value is less than or close to $0.005 \mathrm{~m} \mathrm{~s}^{-1}$ at position X1 (Figure 9a). In contrast, there is more variation at position X2 where this value can be as large as $0.0086 \mathrm{~m} \mathrm{~s}^{-1}$ (Figure 9b). The mean value of all shear velocities (excluding the non-rotating case) at the straight section is ${\overline{U^{*}}}_{\text {str }}=0.0039 \mathrm{~m} \mathrm{~s}^{-1}$ with a standard deviation of $0.0011 \mathrm{~m} \mathrm{~s}^{-1}$. In contrast the shear velocities are almost $18 \%$ larger at the bend apex, where $\bar{U}_{\text {apex }}^{*}=0.0046$ with a standard deviation of $0.0018 \mathrm{~m} \mathrm{~s}^{-1}$. This is despite the overall downstream deceleration between the straight section and the second bend. 

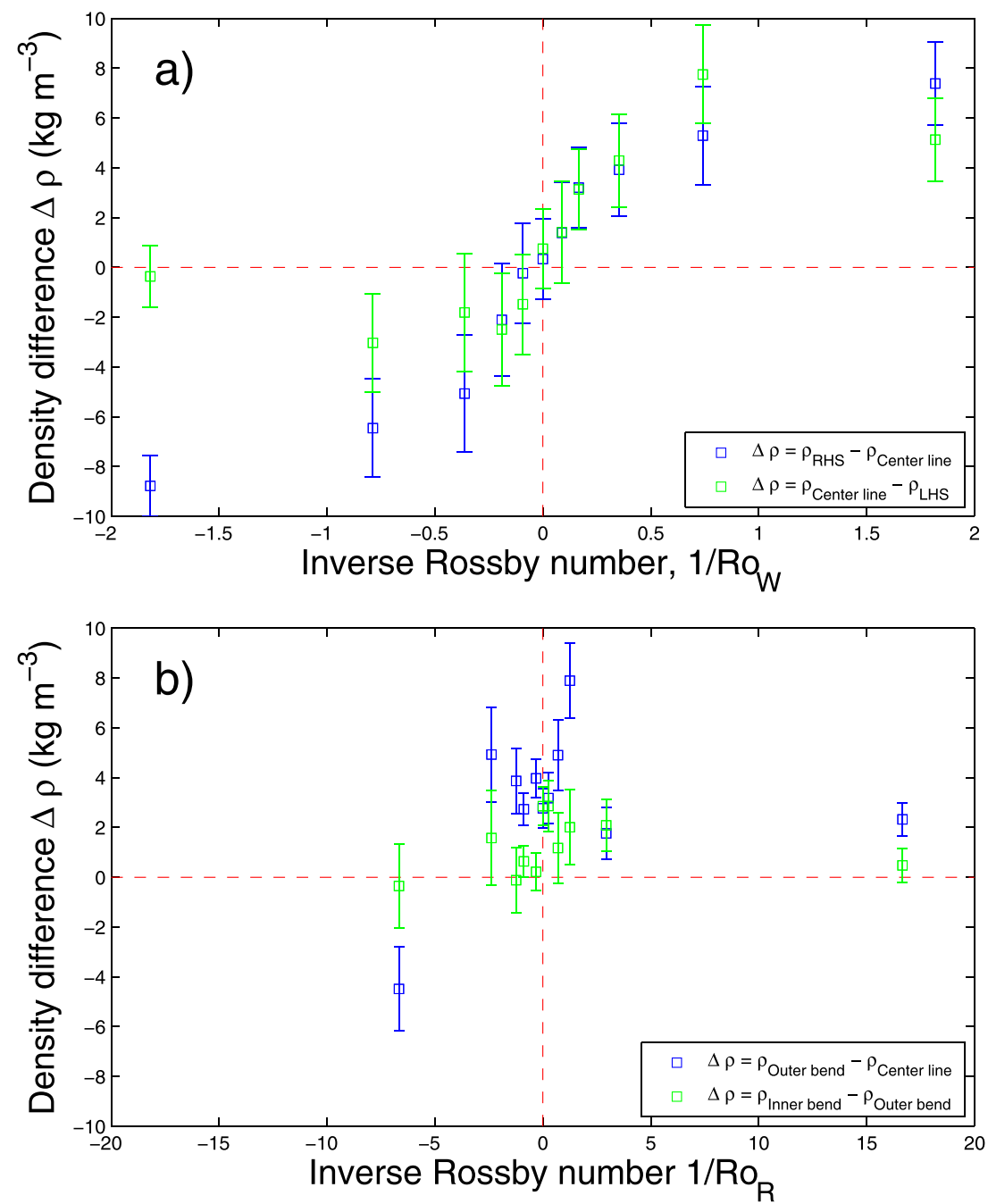

Figure 8. Time-averaged lateral density difference at the (a) straight and (b) bend apex sections as a function of rotation. Density measurements are made at the basal point located $0.044 \mathrm{~m}$ above the floor with the MSTCI conductivity probe. Error bars represent a standard deviation in density measurements.

The drag coefficients, $C_{D}$, at the bend apex are up to two times larger than the straight section (Figures $9 \mathrm{c}$ and 9d), which is in agreement with results from Blanckaert (2010), who found larger drag coefficients in river channel bends than in straight sections. Almost all drag coefficients at the straight section (Figure 9c) are of the order of $5 \times 10^{-4}$. The anomalous points, such as $1 / R o_{W}=-1.82\left(R o_{W}=-0.55\right)$ at the right-hand side position and $1 / R o_{W}=+1.82\left(R o_{W}=+0.55\right)$ at the left-hand side position, are both for cases where the current is completely deflected to one side of the channel, and hence, it is difficult to reliably estimate the downstream velocity to normalize shear velocity. The calculated values for the drag coefficient at this section are consistent in magnitude with previous studies (Cossu et al., 2010; Kessel \& Kranenburg, 1996; Peters \& Johns, 2005). However, drag coefficients at the bend apex are up to two times larger than those at the straight section (Figure $9 \mathrm{~d})$. Note that the drag coefficient data corresponding to $1 / R o_{R}=-6.66\left(R o_{R}=-0.15\right)$ in the outer bend position and $1 / R o_{R}=+16.66\left(R o_{R}=+0.06\right)$ in the inner bend position are not included in Figure $9 \mathrm{~d}$. As the rotation rates were high in these cases, the flow was completely deflected to one side of the channel, and therefore, the collected data were outliers. The mean of all drag coefficients (excluding the nonrotating case) at the straight section is ${\overline{C_{D}}}_{\text {str }}=4.24 \times 10^{-4}$ with the standard deviation of $2.5 \times$ $10^{-4}$. The drag coefficients (excluding the data noted above) are larger on average at the bend $\left(\overline{C_{D}}\right.$ apex $=$ $8.01 \times 10^{-4}$ with a standard deviation of $7.87 \times 10^{-4}$ ), than in the straight section, due to the larger values of shear velocity at the bend. The increased drag coefficients measured at the bend apex suggests that 

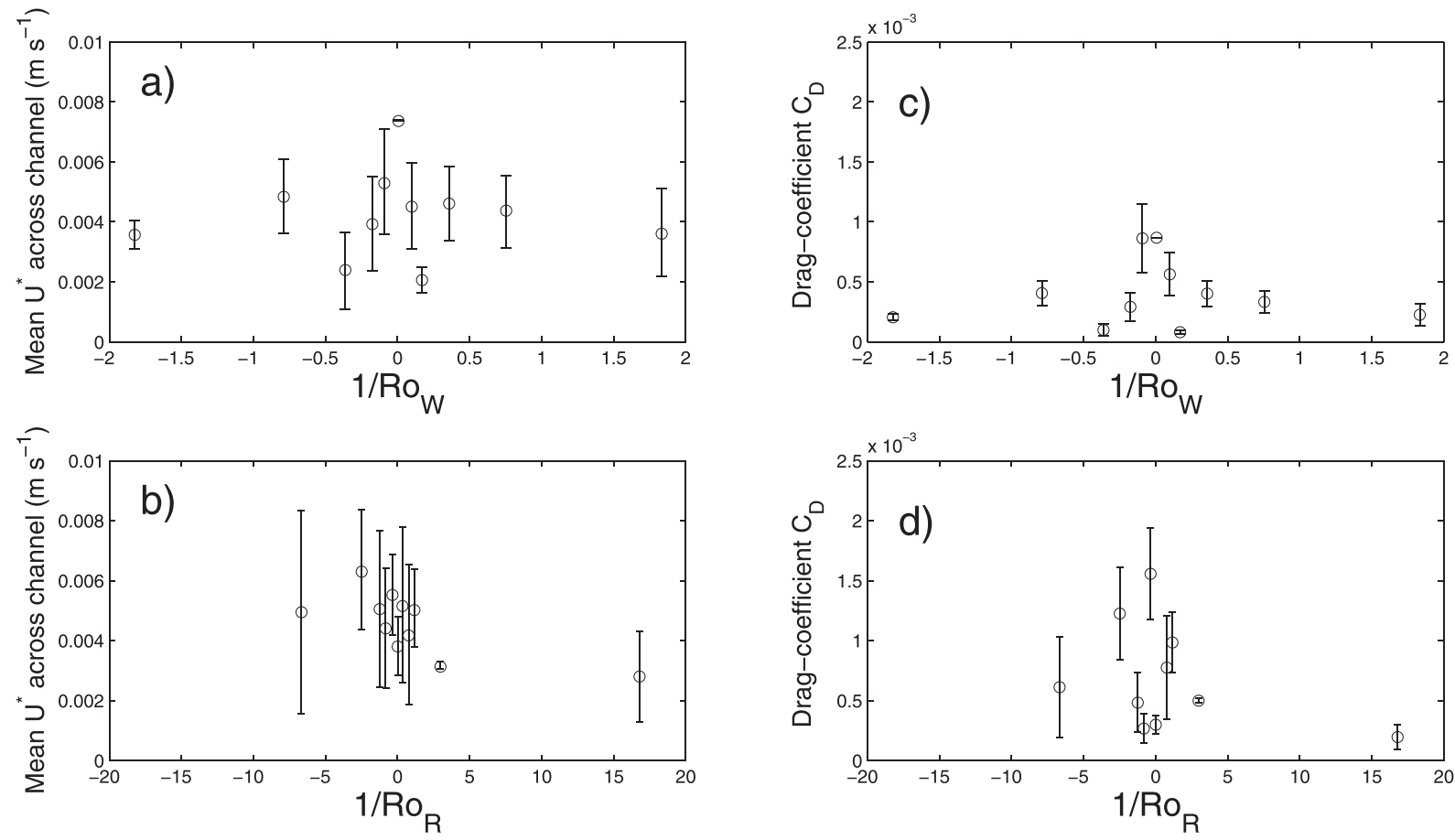

Figure 9. Depth average of shear velocity $U^{*}$ (below $U_{\max }$ ) calculated over three measurement locations across the channel at the (a) straight and (b) bend apex sections as a function of rotation. Mean drag coefficient $\left(C_{D}\right)$ calculated over three measurement locations across the channel as a function of rotation at (c) the straight and (d) the bend apex sections. The bars show the standard deviation for each Rossby number.

there could be more potential for erosion at the bend apex, despite the large-scale deceleration between the straight section and the second bend apex.

\subsection{Ekman Numbers}

The magnitude of the Ekman number can be used to estimate the degree to which the well-mixed bottom boundary layer is dominated by Coriolis $(E k<<1)$ or frictional forces $(E k>>1)$. The absolute values of the inverse Ekman number for different rotation rates are plotted for both the straight and the bend apex sections in Figure 10. Recalling equation (7), the Ekman number can be interpreted as the ratio of the theoretical Ekman boundary layer thickness $d$, to the depth $h$ of the well-mixed layer below the velocity maximum of a gravity current. The Ekman number (see equation (5)) of a well-mixed bottom boundary is approximately unity only in a few rapidly rotating experiments when $0.7<\left|1 / R o_{W}\right|<2.5\left(0.4<\left|R o_{W}\right|<\right.$ 1.4) at the straight section, and when $\left|1 / R o_{R}\right|>2.85\left(\left|R o_{R}\right|<0.35\right)$ at the bend apex. For all other experiments, the Ekman numbers are much larger than one, so that $1 / E k<<1$, implying $d>>h$ (Figure 10). The Ekman number plotted in Figure 2008 gives an idea of how much of the well-mixed boundary is dominated by the Coriolis force versus the frictional force. Classical Ekman theory strictly applies for $E k<<1$. The whole well-mixed boundary layer is a balance of the turbulent (or viscous) shear and the Coriolis force for $E k \sim 1$, while frictional force dominates within the boundary for $E k>>1$, and we might expect to see a classical logarithmic boundary layer. The key result of Figure 10 is that in almost all experiments we have $E k>>1$, so while the Coriolis force is important, we would not expect to see a classic Ekman spiral in the velocity structure of the bottom boundary layer. There is a gradual and continuous increase in Ekman number with a decrease in the rate of rotation for both sections (Figure 10). This implies that there is a gradual transition between the relative importance of the Coriolis force versus frictional force, rather than a sudden sharp transition where frictional forces suddenly dominate. Hence, Coriolis force can still be somewhat important even for $1 / R o_{R}<1\left(R o_{R}>1\right)$. In the straight section (Table $\left.\mathrm{S} 2\right)$, the absolute values of Ekman number are in the range of $1.2<|E k|<12.5$ with only one exception in the case of slowly rotating $R o_{W}=-10.85$ at the centerline, yielding an absolute Ekman number, $|E k|$, with the highest value of 64.37. The value of the inverse Ekman number for all rotation rates in Figure 10a is $0<1 /|E k|<1.2$. On the other 

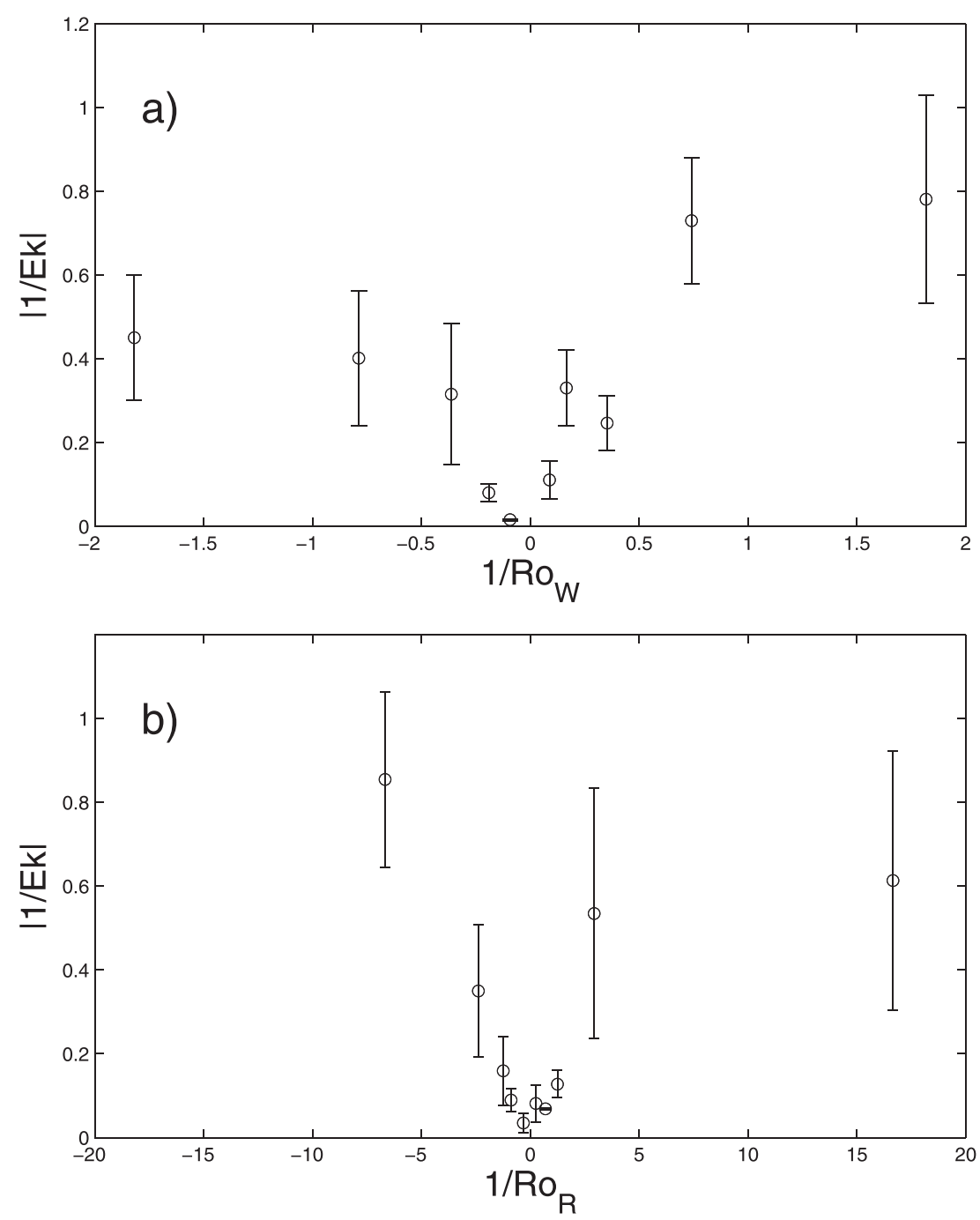

Figure 10. Variations of $1 / E k$ as a function of rotation in (a) the straight and (b) the bend apex section. In almost all cases $|E k|>>1$, except for the most rapidly rotating experiments, where $E k \sim 1$. This implies that the depth of Ekman boundary layer $d$ is much larger than the depth of the well-mixed bottom boundary $h$ that exists below the velocity maxima. As Ek >> 1 in almost all experiments, it is not expected to observe a classical Ekman spiral in the velocity structure of the bottom boundary layer. In this plot only the centerline values of $U^{*}$ are used to estimate $E k$.

hand, $|E k|$ becomes slightly larger at the bend apex and varies within the larger range of $1.1<|E k|<28.8$ (Table S2). This leads to $0<1 /|E k|<1$ for all rotation rates in Figure 10b.

\subsection{Summary of Velocity and Density Variations in the Straight and Curved Channels}

A schematic summary of the relationship between the lateral distribution of velocity and density, as well as interfacial tilt for different Rossby numbers in the straight section of the channel, is shown in Figure 11. These schematics of the behavior of the current and deflection of the density interface use data from experiments with $R o_{W}=\infty,+2.83$ and -2.76 . Our velocity and density data for the nonrotating case (Figure 11a) show relatively small lateral variation, with a small circulation near the walls and no deflection of the interface towards either side of the channel. Fast positive rotation rates (Figure 11b) also show very little variation in downstream velocities on the left-hand side of the channel. Furthermore, the effect of the Coriolis force sends the bulk of the current to the right-hand side of the channel when looking downstream (simulating the Northern Hemisphere), and the interface tilts laterally upwards towards the right-hand wall. When the rotation is reversed (Figure 11c), the density interface is a mirror image of Figure 11b, and downstream velocities on the right-hand side decrease, while the Coriolis force deflects the bulk of the flow and the density 

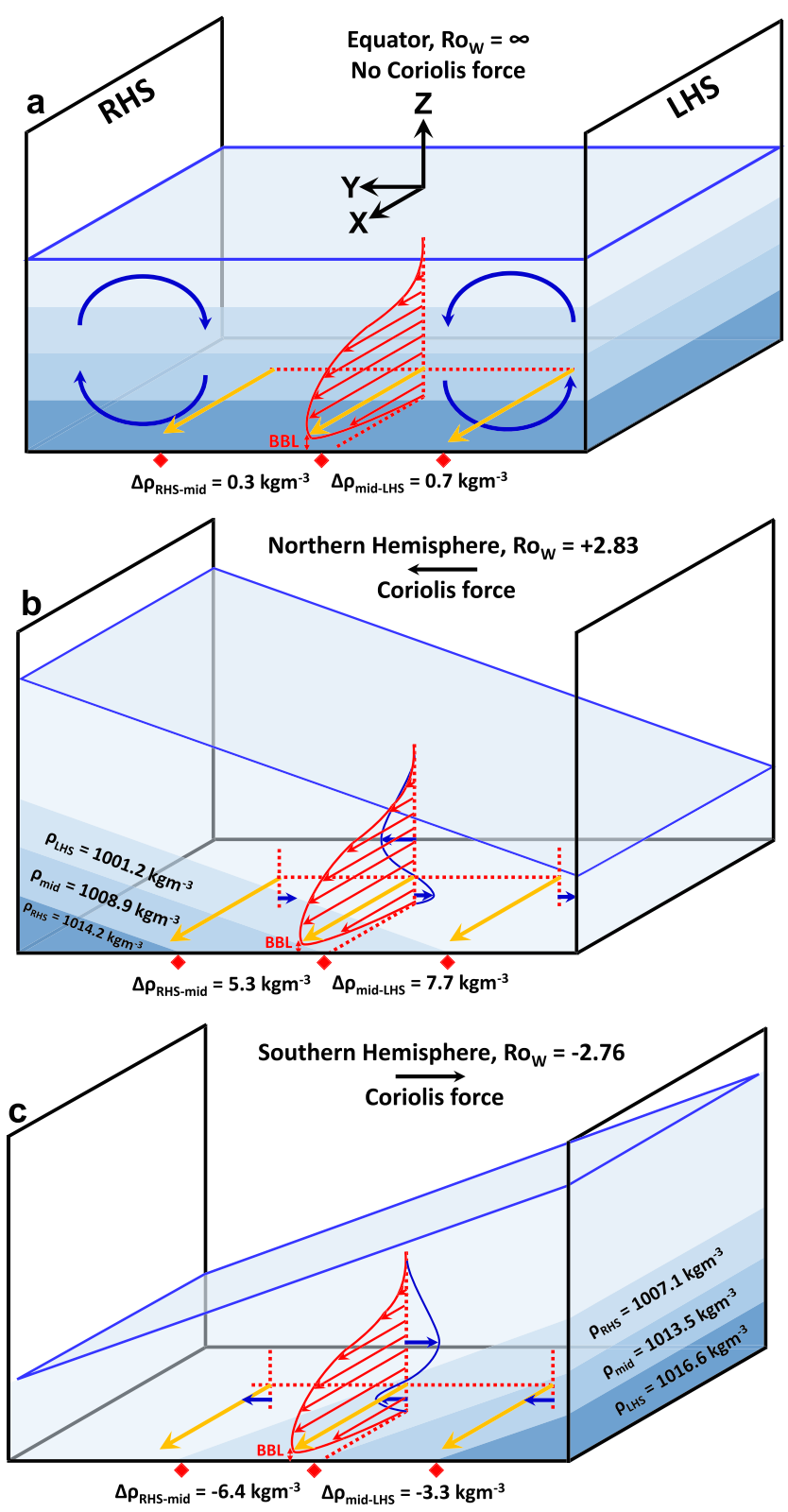

Figure 11. Schematic of the current at the straight section for (a) $R o_{W}=\infty$ (experiment $6 \mathrm{X} 1 \mathrm{EQ}$ ), (b) $R o_{W}=+2.83$ (experiment $3 \mathrm{X} 1 \mathrm{~N}$ ), and (c) $R o_{W}=-2.76$ (experiment 3X1S). Red, yellow, and blue vectors represent the downstream, maximum downstream, and cross-stream velocities, respectively. BBL, bottom boundary layer, defined as the depth below the velocity maximum. The red dashed line indicates where the cross-stream velocity changes direction. Shades of blue schematically illustrate the changes in density with depth, albeit these likely exhibit a more continuous stratification, rather than the simple layers shown herein.

maximum to the left wall. The above observations, of lateral tilt of the upper surface and flow velocities, are in agreement with the experiments of Cossu et al. (2010); however, here we combine these data with detailed measurements of internal density for the first time.

The presence of a bend in the channel changes the circulation pattern of the gravity current and the deflection of the density interface substantially. These changes in the apex section are summarized schematically in Figure 12 for $R o_{R}=\infty,+0.8$, and -0.81 . One of the surprising results this figure highlights is that, while the velocity maxima and upper density interface shift from left to right, the maximum density is always located at the outer bank irrespective of the sense of rotation. In contrast to the straight channel, when maximum density switches sides with the sense of rotation, this suggests a decoupling of the velocity and density fields, 

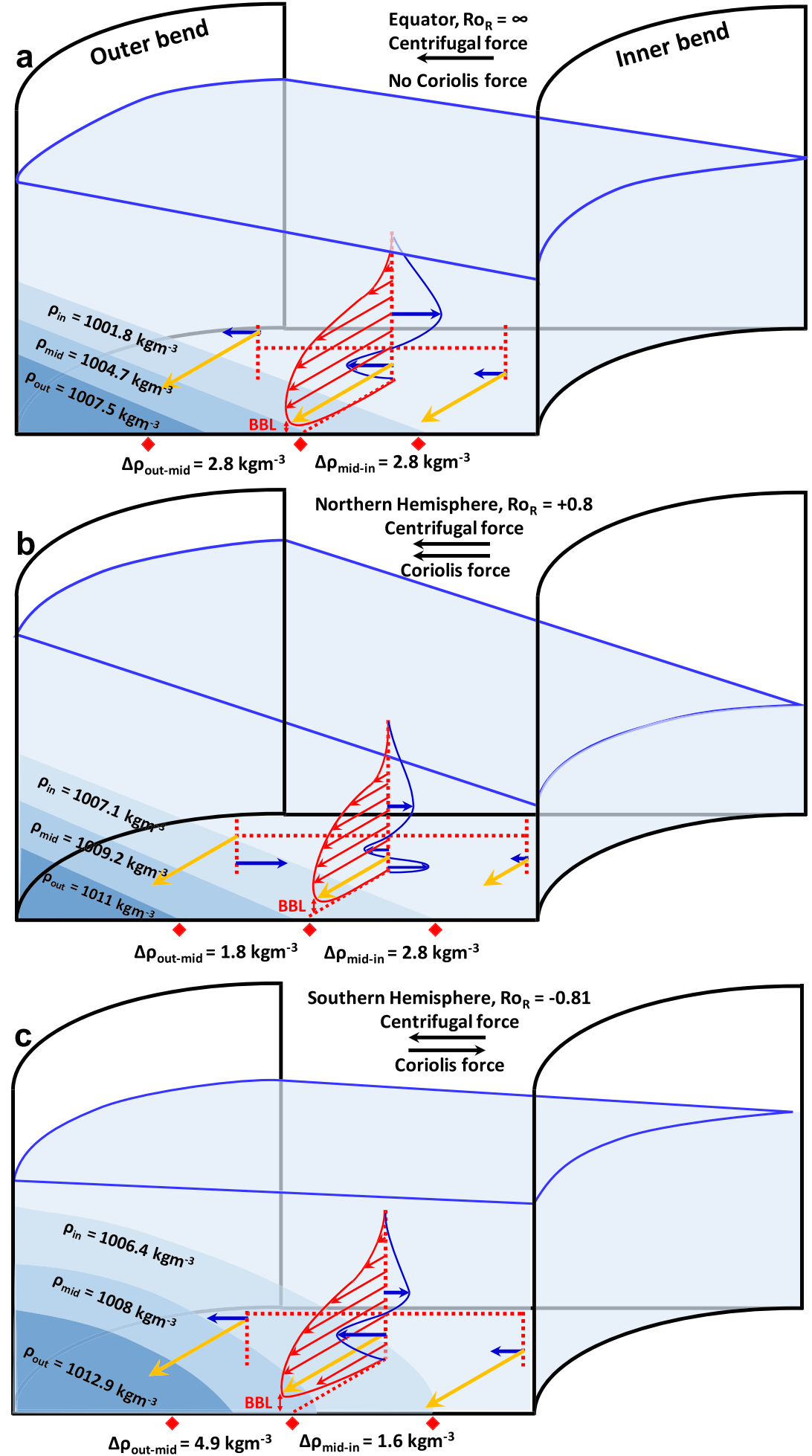

Figure 12. Schematic of the current at the second bend apex section for $(\mathrm{a}) R o_{R}=\infty$ (experiment $\left.6 \mathrm{X} 2 \mathrm{EQ}\right),(\mathrm{b}) R o_{R}=+0.8($ experiment $3 \mathrm{X} 2 \mathrm{~N})$, and $(\mathrm{c}) R o_{R}=-0.81$ (experiment 3X2S). Red, yellow, and blue vectors represent the downstream, maximum downstream, and cross-stream velocities, respectively. Apart from the outer bend measurement position in (a), where the downstream velocity maximum is not recorded in the observation window, thus the vector represents the highest velocity observed. BBL, bottom boundary layer, defined as the depth below the velocity maximum. The red dashed line indicates where the cross-stream velocity changes direction. Shades of blue schematically illustrate the changes in density with depth, albeit these likely exhibit a more continuous stratification, rather than the simple layers shown herein. 
when the Coriolis force opposes the centrifugal force (Figure 13c). In the absence of rotation $\left(R o_{R}=\infty\right.$, simulating a left-turning bend at the Equator), small lateral density gradients and larger downstream velocities at the outer bend are observed, as the centrifugal force preferentially directs the lower part of the flow around the maximum velocity (i.e., that part with most momentum) towards the outer bend (Figure 12a), which is consistent with earlier studies (Cossu \& Wells, 2010; Keevil et al., 2006; Serchi et al., 2011). In the case when the Coriolis and centrifugal forces act together (Figure 12b), corresponding a left-turning bend in the Northern Hemisphere, the core part of the gravity current shifts towards the outer bank. However, the basal part of the flow $(<0.028 \mathrm{~m})$ exhibits secondary flow towards the inner bank, opposite to the direction of the centrifugal force, representing a stratification-modified Ekman boundary layer (see Figures 4e, 6d-6f, and 7d). Similarly, there is also a return flow towards the inner wall at the top of the current. The maximum downstream velocities in the basal part of the flow are characterized by slower values, especially at the inner bend (Figures $5 \mathrm{f}$ and $12 \mathrm{~b}$ ). In contrast, in the case when the centrifugal and Coriolis forces are in opposition (Figure 12c), corresponding to a left-turning bend in the Southern Hemisphere, downstream velocities are more evenly distributed across the width (the three y locations) of the channel (Figures $5 \mathrm{~d}-5 \mathrm{f}$ and $13 \mathrm{c}$ ). Faster downstream velocities are observed in the basal part of the flow and secondary flow is directed towards the outer bank. A clear trend is observed in the basal zone of the flow, with progressive increases in maximum downstream flow velocities with decreased negative Rossby numbers, and a decline in downstream velocities with larger positive Rossby numbers (Figure 7c).

\subsection{Discussion}

3.8.1. Velocity, Density, and Bottom Boundary Layer of the Gravity Current

The experiments described herein highlight new effects of the Coriolis force upon the velocity and density structure of channelized gravity currents. A key result is the observation of a stratification-modified Ekman boundary layer, in left-turning bends where $R o_{R}<+0.8$ (Figures $4 \mathrm{e}, 6 \mathrm{~d}-6 \mathrm{f}$, and $7 \mathrm{~d}$ ). Flow in this basal layer overcomes the dominance of centrifugal effects and is therefore oriented towards the inner bank, rather than the usual observation of flowing away from the inner bank. Consequently, in a sediment-laden flow any bedload sediment transport will have a component oriented towards the inner bank at the bend apex, even though both the centrifugal and Coriolis forces are oriented towards the outer bank. This contrasts with submarine channel flows with the same sense of the centrifugal force, but where the Coriolis force is zero, in which bedload has an outward-directed component at the bend apex (Amos et al., 2010; Dorrell et al., 2013; Keevil et al., 2006). The observed stratification-modified Ekman boundary layer, $d$, is approximately $28-\mathrm{mm}$ thick in the present experiments and constitutes that part of the flow below and immediately above the downstream velocity maximum (Figures $4 \mathrm{~d}$ and $4 \mathrm{e}$ ).

In curved channels, we observed increased downstream velocities in the bottom boundary layer around bends with negative Rossby numbers (Figures $5 \mathrm{~d}-5 \mathrm{f}$ and $7 \mathrm{c}$ ), and this was also reflected in the head velocities of gravity currents with negative Rossby numbers (Figure 3). However, these higher velocities in the basal layer are compensated by lower velocities at height within the current (Figure 4d). Furthermore, we noted an almost doubling of the drag coefficient in the channel bend, compared to the straight section (Figure 10).

Another key observation is that the deflection of the density core was always towards the outside of the channel bend (Figure 8b). This new result is somewhat surprising as it suggests that the internal density structure behaves differently to the upper surface. Previous theory and observations (Cossu \& Wells, 2010; Komar, 1969; Wells \& Cossu, 2013) have all described the tilt of the upper interface in terms of Coriolis and centrifugal forces and shown that this upper surface can tilt towards either the outer or inner bend depending upon the relative strength of Coriolis force. While we do not have a theory as to why the internal density field appears to be decoupled from the velocity field, a number of previous observations of nonrotating and rotating gravity currents are consistent with our observations, suggesting it is an important topic for future research. For flows with no rotational influence $\left(R o_{R}=\infty\right)$ or positive Rossby numbers, the velocity and density maxima are co-located, but when the Coriolis force opposes the centrifugal force (Figure 13c, in the case of $R o_{R}=-0.81$ as well as -0.42 ), the velocity maximum is located towards the inner bank, thus indicating a decoupling of the velocity and density fields. This decoupling is in agreement with that observed in the numerical simulations of a right-turning curved channel with no rotational influence of Serchi et al. (2011; their Figures 13C and 16), with the density maximum located 

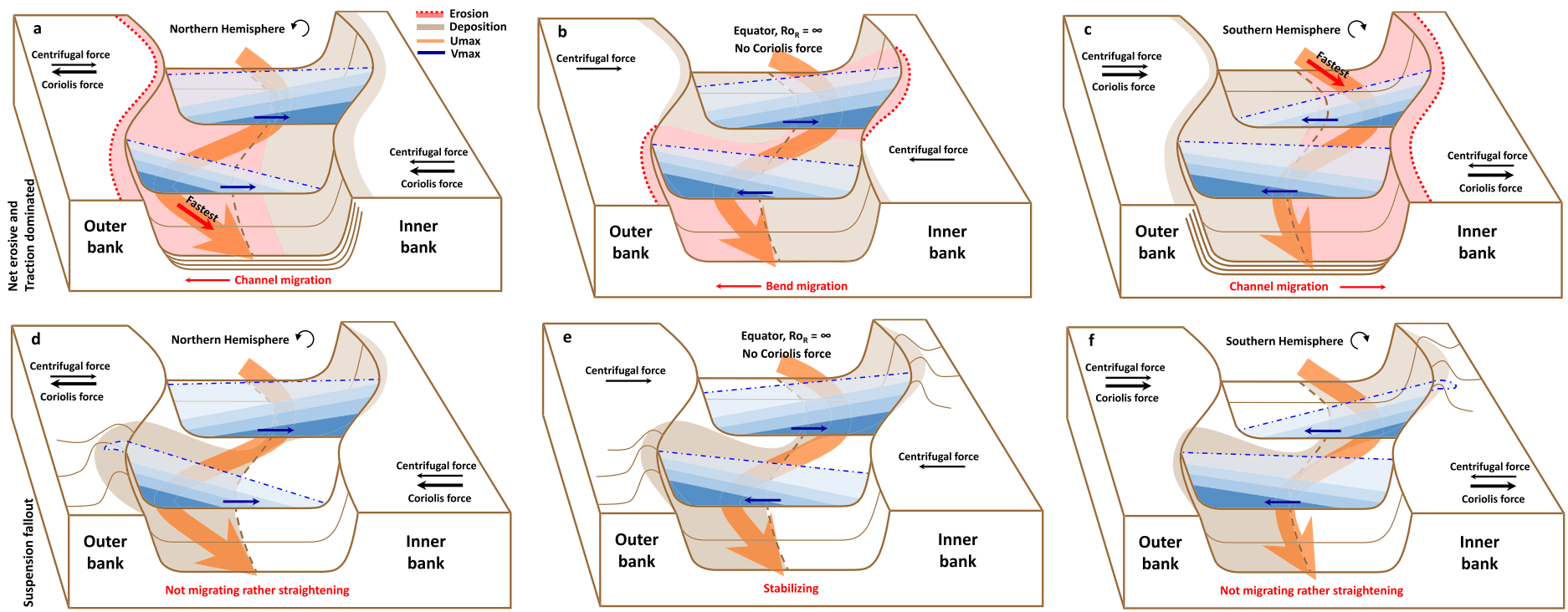

Figure 13. A schematic display of the postulated patterns of erosion and deposition and the evolution of channel sinuosity under the effects of (a-c) traction-dominated and (d-f) suspension fallout transport regimes in the Northern Hemisphere, Equator, and Southern Hemisphere.

towards the outer bank at the bend apex, and the velocity maximum located towards the inner bank. This decoupling of the density and velocity fields has also been observed in the Black Sea subaqueous channel (Sumner et al., 2014), although in this case with an estimated $R o_{R}$ of +0.87 , and by Wells and Cossu (2013) in a left-turning Northern Hemisphere bend, where the density maximum was found at the inner bend, whereas the velocity maximum was found at the outer bend. Consequently, the sense of decoupling was opposite to the observations from our experimental cases at equivalent negative Rossby numbers. No decoupling of the velocity and density fields was observed for positive rotations in the present experiments. Similar results to those of Sumner et al. (2014), with decoupling and the location of the density maximum shifting towards the inner bend, have been found in analogous systems such as stratified flows in curved large oceanographic straits (Umlauf et al., 2007; Umlauf \& Arneborg, 2009a, 2009b) in highly stratified curved estuarine flows (Nidzieko et al., 2009; Seim \& Gregg, 1997) and in a simulation of a sinuous submarine channel in the absence of Coriolis force (Janocko et al., 2013). While not displaying lateral variation in downstream velocities, the numerical simulations of curved channels without the influence of Coriolis force by Ezz and Imran (2014) mainly showed outer bank density maxima but demonstrated that the density maximum can change to the inner bank simply by increasing the channel width, even though the sense of secondary circulation remained the same. The present results suggest that these differences in the nature of flow decoupling are the product of the Coriolis force, along with flow stratification, bend geometry, slope, and flow discharge (Ezz \& Imran, 2014; Sumner et al., 2014). Collectively, these processes will influence the phase lag between the secondary current strength and channel curvature (Ezz \& Imran, 2014), in turn potentially controlling when and how density and velocity decoupling occurs.

\subsubsection{Implications for Channel Evolution}

In the present experiments, we have measured flow at a single bend apex. However, in this case, we have varied Rossby number so that the effects of both left- and right-turning bends on the flow can be seen in terms of variations between positive and negative Rossby numbers. Thus for a Northern Hemisphere channel, positive Rossby numbers relate to left-turning bends, and negative Rossby numbers relate to right-turning bends. The new results from our saline gravity current experiments are potentially important for understanding transport and erosion in dynamically similar turbidity currents constrained to sinuous submarine channels. In the following section, we will attempt to understand how changes in density and velocity structure due to the Coriolis force may influence the location of erosion and deposition with channels, and hence, how channel sinuosity could evolve with time. 
Two new results from our experiments are especially important for the temporal evolution of channel sinuosity at high latitudes. The first result is the asymmetry in near-bed downstream velocity between left- and right-turning bends (Figure 7c), and the second is the observation that the density core is always deflected to the outside of the bend (Figure $8 \mathrm{~b}$ ). As the velocity maximum is located on the inside of the left-turning bend when the Coriolis force is large and negative (see Figure 3), this implies there is much greater potential for erosion to occur in a way that diminishes the tendency for channels to become sinuous. One of the surprising results summarized in Figure 12 is that even though the position of the velocity maximum shifted laterally Coriolis force changed, the maximum density is always located at the outside of the channel bend. Our experiment used salt, but if this was a turbidity current then the largest density would correspond to the region of highest sediment load, which in turn would influence where deposition occurred.

Our results demonstrate that the Coriolis force, even at moderate Rossby numbers, leads to significant velocity and density asymmetries between left- and right-turning bends. This could influence the growth of submarine channel sinuosity at midlatitudes, where many flows in channels have $\left|R o_{R}\right|<10$ (Wells \& Cossu, 2013). The motivation for doing these large-scale rotating experiments was to test if they offered a potential explanation for the field observation of Peakall et al. (2012) that higher latitude submarine channels are generally straighter than those at the Equator. Peakall et al. (2012) postulated that the Coriolis force might explain a possible asymmetry between the development of left- and right-turning channel bends, but at the time, they did not elucidate a convincing mechanism. Cossu et al. (2015) subsequently proposed that submarine channel development might depend upon latitude due to shifts in the lateral location of the downstream velocity core under the effect of the Coriolis force. Sinuous channels could then have an asymmetry in the flow dynamics between left- and right-turning bends, where if the velocity core is laterally deflected bends on one side of the channel would stop growing, therefore restricting sinuosity. This explanation of Cossu et al. (2015) linked the measurements of bed dynamics and the flow. However, the size of their experimental setup hindered the acquisition of detailed basal flow measurements and was too small to assess the development of Ekman boundary layers.

The new contribution of the current study is based upon measurements of internal velocity, density structure, and near-bed turbulence. We have synthesized our new findings on velocity, density, and turbulence structure in gravity currents to make a schematic diagram of the possible implications with respect to the evolution of channel sinuosity at different latitudes in Figure 13. In this figure, we postulate how the changes in downstream and cross-stream velocities and density structure of these saline gravity currents could influence large-scale sediment-laden turbidity currents. Here, we examine two styles of sedimentation from a turbidity current, net erosive and traction dominated (Figures 13a-13c), and suspensiondominated (Figures 13d-13f). In the net erosive and traction-dominated case, sediment movement is controlled by a combination of the locus of downstream velocity maximum and by secondary flow in the bottom boundary layer, which includes stratification-modified Ekman boundary layers (where present). In the suspended sediment case, sediment fallout is dominated by the flow concentration and thickness. The schematic in Figure 13 illustrates the evolution of channel sinuosity under the effects of traction- and suspension-dominated regimes in the Northern Hemisphere, the Equator, and the Southern Hemisphere. This figure advances the observations of Cossu et al. (2015), as we have now measured the changes in turbulent strength and internal density structure of gravity currents under different rotation rates and at high Reynolds numbers.

In the absence of the Coriolis force in a sinuous channel, the centrifugal force is typically greatest at the level of the maximum downstream velocity. For flows with near-bed secondary circulation directed towards the outer bank at the bend apex as occur herein (Figure 7d), that is, reversed relative to rivers, the locus of maximum downstream velocity is also deflected towards the outer bank (Amos et al., 2010; Dorrell et al., 2018; Keevil et al., 2006; Straub et al., 2008; Wells \& Cossu, 2013). These higher downstream velocities at the outer bank would result in higher erosion potential at outer bend locations in both right- and left-turning bends (Figures 13b and 13e), consequently channel sinuosity would increase with time, in line with the standard descriptions for sinuous channel evolution in both subaerial and subaqueous settings (Ikeda et al., 1981; Imran et al., 1999; Johannesson \& Parker, 1989; Seminara, 2006). Many experimental studies on subcritical and supercritical flows in submarine channel bends at smaller scales (Table S1), report basal secondary flow pointing towards the outer bend, at the bend apex, accompanied by a surface return flow (Corney et al., 2008; 
Cossu \& Wells, 2010; Keevil et al., 2006), similar to our observations in the cross-stream velocity profiles depicted in Figure 4e (black curve representing $R o_{R}=\infty$ ). This outward-directed secondary flow component in the upstream parts of the bend leads to deposition at the inner bend due to the convergence of fluid and sediment being delayed farther around the bend compared to fluvial examples (Darby \& Peakall, 2012; Peakall et al., 2007).

In the suspension fallout phase when there is no rotation, sediment is preferentially deposited at outer bend apices, where the density maximum is located, and fine sediments are over spilled by the superelevation of upper interface centrifugal force (Figure 13e) (Cossu et al., 2015; Kane et al., 2008; Nakajima et al., 2009; Straub et al., 2011). This periodic outer bank deposition is postulated to help in the stabilization of sinuosity in the latter stages of submarine channel evolution at low latitudes (Amos et al., 2010; Kane et al., 2008; Straub et al., 2008).

In the Northern Hemisphere under the net erosive phase, there would be a strong deflection of the flow towards the outside of the left-turning bend, as the centrifugal and Coriolis forces both act in the same direction and the maximum downstream velocity gets even closer to this bend (Figures 13a and 7c). Since the location of maximum velocity controls the location of erosion in net erosive flows, more erosion would likely occur in the outer bank of the left-turning bend as displayed in Figure 13a. On the other hand, the centrifugal and Coriolis forces oppose each other in the right-turning bend. Maximum downstream velocities are very similar at all three positions across the bed, but the height of the maximum downstream velocity is close to the bed at the inner bank and becomes progressively higher across the channel (Figure 13c). Therefore, the basal velocity gradient is much higher at the inner bank, and consequently, bed shear stress will also be much greater, leading to higher potential for erosion and sediment transport at this location, i.e., on the right-hand side of the channel looking downstream. Thus, erosion would likely occur on all of the right-hand side of the channel as illustrated in Figure 13a. In the traction-dominated phase in the Northern Hemisphere, the secondary circulation (usually $10 \%$ of the downstream velocity but can be as large as 50\% in some cases; Keevil et al., 2006, 2007; Pyles et al., 2012) would carry a large amount of eroded sediment downstream of the inner bank of the left-turning bend and into the right-turning bend as illustrated schematically in Figure 13a. The areas of erosion and deposition are mirrored in the Southern Hemisphere (Figure 13c).

The asymmetry in the distribution of erosion and deposition processes at higher latitudes in the Northern Hemisphere in Figure 13a results in a net migration to the right looking downstream and to the left for the Southern Hemisphere case in Figure 13c. More importantly, we suggest that by reducing the tendency for one bend to erode and become more sinuous, these higher latitude channels will not become more sinuous but rather just migrate. Such a shift in erosion pattern could act to stop the growth of initial perturbations (described by Ikeda et al., 1981), which are thought to promote sinuosity. This offers a possible mechanism to explain observations of systematic decreases in submarine channel sinuosity with latitude (Peakall et al., 2012).

The fallout of suspended sediment is most likely to occur at the location of the density maximum of the gravity current, in the Northern Hemisphere this occurs at the outer banks of both bends (Figure 13d). However, there is a thicker flow at the outer bank of the left-turning bend looking downstream as both the Coriolis and centrifugal forces produce a greater deflection in the current, and hence, more sediment is deposited in this area. As there is more deposition at one bend, the channel is not migrating but rather straightening under the effect of suspension fallout in the Northern Hemisphere. In the Southern Hemisphere a mirrored sedimentation pattern is observed as illustrated in Figure 13f.

The tendency for overbanking flows depends upon the superelevation of the upper interface, which shows an asymmetry between channel bends when the Coriolis force becomes important (Figures 13d and 13f). A standard observation is that in the Northern Hemisphere, channel levees are high on the right-hand side (looking downstream) and in the Southern Hemisphere they are higher on the left-hand side (Komar, 1969). In Figure 13d, it is postulated that more sediment is deposited towards the outside of the left-turning bend, where the Coriolis and centrifugal forces create a larger deflection of the density current. Therefore, higher levee heights should be observed on the right-hand side at the left tuning bend. In the Southern Hemisphere on the other hand, such a pattern is mirrored, and there should be higher levee heights at the left-hand side of the right-turning bend. 


\section{Conclusions}

The important new observations in this study show that the Coriolis force has a substantial effect on the location of the density structure and magnitude and direction of internal flows within a saline gravity current flowing along a deep-water channel. The location of the maximum velocity core, and the orientation of the bottom boundary layer, of a gravity current change under the effect of the Coriolis force, which causes left- and right-turning bends to be asymmetric. However, the denser fluid always remains at the outer bend, providing a hydraulic mechanism for stabilizing the evolution of bends at higher latitudes. In particular, strong Coriolis force can flip the direction of the near-bed secondary flow, which is predicted to strongly influence sediment transport in turbidity currents and hence, the evolution of straight and sinuous deepwater channels at different latitudes. Such deep-water channels are critical conduits for deep thermohaline circulation in the ocean and are often important pathways for the transport of sediment by turbidity currents. The use of a rotating 13-m radius channel gave an unprecedented view of high Reynolds number rotating gravity currents, so that this study has by far the most detailed velocity and density measurement of a laboratory channelized gravity current in a controlled environment in both straight and sinuous channels, with and without rotation. To our knowledge, these experiments represent the largest and most turbulent gravity current ever tested under the effect of Coriolis force, allowing a meaningful extrapolation to fieldscale underwater gravity currents in the ocean. Previous studies with much smaller experimental setups (Cossu et al., 2010; Cossu et al., 2015; Cossu \& Wells, 2010; Keevil et al., 2006) and lower Reynolds numbers were not capable of resolving the thin bottom boundary layer. Our results provide an explanation for the effect of the Coriolis force on the velocity structure of the gravity current flowing within a straight channel, as observed in the previous study of Cossu et al. (2010). The symmetric pattern in the downstream and crossstream velocity profiles in our straight channel indicates the symmetry in patterns of circulation between the Northern and Southern Hemispheres.

In our channel bend, the key result is that for small Rossby numbers where $\left|R o_{R}\right|<+0.8$, the Coriolis force dominates the centrifugal force and changes the near-bed flow direction in the bottom boundary layer, through the development of a stratification-modified Ekman boundary layer. This key feature of the secondary flow under the influence of rotation builds upon previous nonrotating studies, where the centrifugal force dominates (Corney et al., 2006, 2008; Cossu \& Wells, 2010; Dorrell et al., 2018; Keevil et al., 2006). Furthermore, the velocity and density fields in sinuous channels are shown to be decoupled. The Coriolis force moves the location of the maximum downstream velocity core laterally to the left or right depending on the sense of rotation (i.e., Northern or Southern Hemisphere), while the centrifugal force always sends the denser portion of the current towards the outer bend of the channel. We synthesize these observations into a new conceptual model of the underlying flow mechanics of sinuous turbidity currents and resultant sedimentation that explains the nature of observed sinuosity variations at both low and high latitudes.

\section{References}

Abad, J. D., Sequeiros, O. E., Spinewine, B., Pirmez, C., Garcia, M. H., \& Parker, G. (2011). Secondary current of saline underflow in a highly meandering channel: experiments and theory. Journal of Sedimentary Research, 81, 787-813. https://doi.org/10.2110/jsr.2011.61

Akhmetzhanov, A., Kenyon, N. H., Habgood, E., Van DerMollen, A. S., Nielsen, T., Ivanov, M., \& Shashkin, P. (2007). North Atlantic contourite sand channels. In A. R. Viana, \& M. Rebesco (Eds.), Economic and palaeoceanographic significance of contourite deposits, Geolo. Soc. London Spec. Pub. (Vol. 276, pp. 25-47). London, UK: Geological Society of London.

Amos, K. J., Peakall, J., Bradbury, P. W., Roberts, M., Keevil, G., \& Gupta, S. (2010). The influence of bend amplitude and planform morphology on flow and sedimentation in submarine channels. Marine and Petroleum Geology, 27, 1431-1447. https://doi.org/10.1016/j. marpetgeo.2010.05.004

Arneborg, L., Fiekas, V., Umlauf, L., \& Burchard, H. (2007). Gravity current dynamics and entrainment-A process study based on observations in the Arkona Basin. Journal of Physical Oceanography, 37, 2094-2113. https://doi.org/10.1175/JPO3110.1

Benedict, L. H., \& Gould, R. D. (1996). Towards better uncertainty estimates for turbulence statistics. Experiments in Fluids, 22(2), $129-136$.

Blanckaert, K. (2010). Topographic steering, flow recirculation, velocity redistribution, and bed topography in sharp meander bends. Water Resources Research, 46, W09506. https://doi.org/10.1029/2009WR008303

Caldwell, D. R., Van Atta, C. W., \& Helland, K. N. (1972). A laboratory study of the turbulent Ekman layer. Geophysical Fluid Dynamics, 3 , $125-160$.

Cenedese, C., \& Adduce, C. (2008). Mixing in a density-driven current flowing down a slope in a rotating fluid. Journal of Fluid Mechanics, 604, 369-388.

Cenedese, C., Whitehead, J. A., Ascarelli, T. A., \& Ohiwa, M. (2004). A dense current flowing down a sloping bottom in a rotating fluid. Journal of Physical Oceanography, 34(1), 188-203.

Corney, R. K. T., Peakall, J., Parsons, D. R., Elliott, L., Amos, K. J., Best, J. L., et al. (2006). The orientation of helical flow in curved channels. Sedimentology, 53, 249-257. 
Corney, R. K. T., Peakall, J., Parsons, D. R., Elliott, L., Best, J. L., Thomas, R. E., et al. (2008). Reply to discussion of Imran et al. on “The orientation of helical flow in curved channels" by Corney et al., Sedimentology, 53, 249-257. Sedimentology, 55, $241-247$.

Cossu, R., \& Wells, M. G. (2010). Coriolis forces influence the secondary circulation of gravity currents flowing in large-scale sinuous submarine channel systems. Geophysical Research Letters, 37, L17603. https://doi.org/10.1029/2010GL044296

Cossu, R., \& Wells, M. G. (2012). A comparison of the shear stress distribution in the bottom boundary layer of experimental density and turbidity currents. European Journal of Mechanics - B/Fluids, 32, 70-79.

Cossu, R., \& Wells, M. G. (2013). The evolution of submarine channels under the influence of Coriolis forces: Experimental observations of flow structures. Terra Nova, 25(1), 65-71.

Cossu, R., Wells, M. G., \& Peakall, J. (2015). Latitudinal variations in submarine channel sedimentation patterns: The role of Coriolis forces. Journal of the Geological Society of London, 172, 161-174.

Cossu, R., Wells, M. G., \& Wåhlin, A. K. (2010). Influence of the Coriolis force on the velocity structure of gravity currents in straight submarine channel systems. Journal of Geophysical Research, 115, C11016. https://doi.org/10.1029/2010JC006208

Cushman-Roisin, B. (1994). Introduction to geophysical fluid dynamics. Upper Saddle River, N. J.: Prentice-Hall.

Dallimore, C. J., Imberger, J., \& Ishikawa, T. (2001). Entrainment and turbulence in saline underflow in Lake Ogawara. Journal of Hydraulic Engineering, 127(11), 937-948.

Darby, S. E., \& Peakall, J. (2012). Modelling the equilibrium bed topography of submarine meanders that exhibit reversed secondary flows. Geomorphology, 163-164, 99-109. https://doi.org/10.1016/j.geomorph.2011.04.050

Darelius, E. (2008). Topographic steering of dense overflows: Laboratory experiments with V-shaped ridges and canyons. Deep-Sea Research Part I: Oceanographic Research Papers, 55(8), 1021-1034.

Darelius, E., \& Wåhlin, A. (2007). Downward flow of dense water leaning on a submarine ridge. Deep-Sea Research Part I: Oceanographic Research Papers, 54(7), 1173-1188.

Davies, P. A., Wahlin, A. K., \& Guo, Y. (2006). Laboratory and analytical model studies of the Faroe Bank Channel deep-water outflow. Journal of Physical Oceanography, 36, 1348-1364. https://doi.org/10.1175/JPO2917.1

Dorrell, R. M., Darby, S. E., Peakall, J., Sumner, E. J., Parsons, D. R., \& Wynn, R. B. (2013). Superelevation and overspill control secondary flow dynamics in submarine channels. Journal of Geophysical Research, Oceans, 118(8), 3895-3915. https://doi.org/ 10.1002/jgrc. 20277

Dorrell, R. M., Peakall, J., Burns, C., \& Keevil, G. M. (2018). A novel mixing mechanism in sinuous seafloor channels: Implications for submarine channel evolution. Geomorphology, 303, 1-12.

Dorrell, R. M., Peakall, J., Sumner, E. J., Parsons, D. R., Darby, S. E., Wynn, R. B., et al. (2016). Flow dynamics and mixing processes in hydraulic jump arrays: Implications for channel-lobe transition zones. Marine Geology, 381, 181-193.

Ellison, T. H., \& Turner, J. S. (1959). Turbulent entrainment in stratified flows. Journal of Fluid Mechanics, 6, 423-448.

Ezz, H., \& Imran, J. (2014). Curvature-induced secondary flow in submarine channels. Environmental Fluid Mechanics, 14, $343-370$.

Fer, I., Voet, G., Seim, K. S., Rudels, B., \& Latarius, K. (2010). Intense mixing of the Faroe Bank Channel overflow. Geophysical Research Letters, 37, L02604. https://doi.org/10.1029/2009GL041924

Ferrero, E., Longhetto, A., Montabone, L., Mortarini, L., Manfrin, M., Sommeria, J., et al. (2005). Physical simulations of neutral boundary layer in rotating tank. Nuovo Cimento, 28, 1-17.

Garrett, C., MacCready, P., \& Rhines, P. (1993). Boundary mixing and arrested Ekman layers: Rotating stratified flow near a sloping boundary. Annual Review of Fluid Mechanics, 25(1), 291-323.

Gessner, F. B. (1973). The origin of secondary flow in turbulent flow along a corner. Journal of Fluid Mechanics, 58, 1-25.

Gong, C., Peakall, J., Wang, Y., Wells, M. G., \& Xu, J. (2017). Flow processes and sedimentation in contourite channels on the northwestern South China Sea margin: A joint 3D seismic and oceanographic perspective. Marine Geology, 393, 176-193.

Gray, T. E., Alexander, J., \& Leeder, M. R. (2006). Longitudinal flow evolution and turbulence structure of dynamically similar, sustained, saline density and turbidity currents. Journal of Geophysical Research, 111, C08015. https://doi.org/10.1029/2005JC003089

Hesse, R., Chough, S. K., \& Rakofsky, A. (1987). The north-west Atlantic mid-ocean channel of the Labrador Sea: V. Sedimentology of a giant deep-sea channel. Canadian Journal of Earth Sciences, 24, 1595-1624.

Howroyd, G. C., \& Slawson, P. (1975). The characteristics of a laboratory produced turbulent Ekman layer. Boundary-Layer Meteorology, $8(2), 201-219$

Ikeda, S., Parker, G., \& Sawai, K. (1981). Bend theory of river meanders. Part 1. Linear development. Journal of Fluid Mechanics, 112, 363-377.

Imran, J., Islam, M., Huang, H., Kassem, A., Pirmez, C., Dickerson, J., \& Parker, G. (2007). Helical flow couplets in submarine gravity underflows. Geology, 35, 659-662. https://doi.org/10.1130/G23780A.1

Imran, J., Parker, G., \& Pirmez, C. (1999). A nonlinear model of flow in meandering submarine and subaerial channels. Journal of Fluid Mechanics, 400, 295-331.

Islam, M. A., \& Imran, J. (2008). Experimental modeling of gravity underflow in a sinuous submerged channel. Journal of Geophysical Research, 113, C07041. https://doi.org/10.1029/2007JC004292

Ivanov, V. V., Shapiro, G. I., Huthnance, J. M., Aleynik, D. L., \& Golovin, P. N. (2004). Cascades of dense water around the world ocean. Progress in Oceanography, 60(1), 47-98.

Janocko, M., Cartigny, M. B. J., Nemec, W., \& Hansen, E. W. M. (2013). Turbidity current hydraulics and sediment deposition in erodible sinuous channels: laboratory experiments and numerical simulations. Marine and Petroleum Geology, 41, 222-249.

Johannesson, H., \& Parker, G. (1989). Secondary flow in mildly sinuous channels. Journal of Hydraulic Engineering, 115, 289-308.

Johnson, G. C., \& Ohlsen, D. R. (1994). Frictionally modified rotating hydraulic channel exchange and ocean outflows. Journal of Physical Oceanography, 24(1), 66-78.

Johnson, G. C., \& Sanford, T. B. (1992). Secondary circulation in the Faroe Bank Channel Outflow. Journal of Physical Oceanography, 22, 927-933.

Kane, I. A., McCaffrey, W. D., \& Peakall, J. (2008). Controls on sinuosity evolution within submarine channels. Geology, 36, 287-290.

Keevil, G., Peakall, J., \& Best, J. L. (2007). The influence of scale, slope and channel geometry on the flow dynamics of submarine channels. Marine and Petroleum Geology, 24, 487-503.

Keevil, G. M., Peakall, J., Best, J. L., \& Amos, K. J. (2006). Flow structure in sinuous submarine channels: Velocity and turbulence structure of an experimental submarine channel. Marine Geology, 229, 241-257. https://doi.org/10.1016/j.margeo.2006.03.010

Kessel, T. V., \& Kranenburg, C. (1996). Gravity current of fluid mud on sloping bed. Journal of Hydraulic Engineering, 122(12), 710-717.

Komar, P. D. (1969). The channelized flow of turbidity currents with application to Monterey deep-sea fan channel. Journal of Geophysical Research, 74(18), 4544-4558. 
Legg, S., Briegleb, B., Chang, Y., Chassignet, E. P., Danabasoglu, G., Ezer, T., et al. (2009). Improving oceanic overflow representation in climate models: The gravity current entrainment climate process team. Bulletin of the American Meteorological Society, 90(5), 657-670.

Maslin, M., Knutz, P. C., \& Ramsay, T. (2006). Millennial-scale sea-level control on avulsion events on the Amazon Fan. Quaternary Science Reviews, 25, 3338-3345.

Meiburg, E., \& Kneller, B. C. (2010). Turbidity currents and their deposits. Annual Review of Fluid Mechanics, 42, 135-156. https://doi.org/ 10.1146/annurev-fluid-121108-145618

Mercado, A., \& Van Leer, J. (1976). Near bottom velocity and temperature profiles observed. Geophysical Research Letters, 3, $633-636$. https://doi.org/10.1029/GL003i010p00633

Muench, R., Padman, L., Gordon, A., \& Orsi, A. (2009). A dense water outflow from the Ross Sea, Antarctica: Mixing and the contribution of tides. Journal of Marine Systems, 77(4), 369-387. https://doi.org/10.1016/j.jmarsys.2008.11.003

Nakajima, T., Peakall, J., McCaffrey, W. D., Paton, D. A., \& Thompson, P. J. P. (2009). Outer-bank bars: A new intra-channel architectural element within sinuous submarine slope channels. Journal of Sedimentary Research, 79, 872-886.

Nezu, I., Tominaga, A., \& Nakagawa, H. (1993). Field measurements of secondary currents in straight rivers. Journal of Hydraulic Engineering, 119, 598-614.

Nidzieko, N. J., Hench, J. L., \& Monismith, S. G. (2009). Lateral circulation in well-mixed and stratified estuarine flows with curvature. Journal of Physical Oceanography, 39, 831-851.

Peakall, J., Amos, K. J., Keevil, G. M., Bradbury, P. W., \& Gupta, S. (2007). Flow processes and sedimentation in submarine channel bends. Marine and Petroleum Geology, 24, 470-486.

Peakall, J., Kane, I. A., Masson, D. G., Keevil, G., McCaffrey, W., \& Corney, R. (2012). Global (latitudinal) variation in submarine channel sinuosity. Geology, 40(1), 11-14. https://doi.org/10.1130/G32295.1

Peakall, J., \& Sumner, E. J. (2015). Submarine channel flow processes and deposits: A process-product perspective. Geomorphology, 244, 95-120.

Peakall, J., Wells, M. G., Cossu, R., Kane, I. A., Masson, D. G., Keevil, G. M., et al. (2013). Global (latitudinal) variation in submarine channel sinuosity: Reply. Geology, 41, e288.

Perlin, A., Moum, J. N., Klymak, J. M., Levine, M. D., Boyd, T., \& Kosro, P. M. (2007). Organization of stratification, turbulence, and veering in bottom Ekman layers. Journal of Geophysical Research, 112, C05S90. https://doi.org/10.1029/2004JC002641

Peters, H., \& Johns, W. E. (2005). Mixing and entrainment in the Red Sea outflow plume. Part II: Turbulen ce characteristics. Journal of Physical Oceanography, 35, 584-600.

Peters, H., \& Johns, W. E. (2006). Bottom layer turbulence in in the Red Sea outflow plume. Journal of Physical Oceanography, 36, 1763-1785. https://doi.org/10.1175/JPO2939.1

Piper, D. J. W., \& Deptuck, M. (1997). Fine-grained turbidites of the Amazon Fan: Facies characterization and interpretation. In R. D. Flood, D. J. W. Piper, A. Klaus, \& L. C. Peterson (Eds.), Proceedings of the Ocean Drilling Program, Scientific Results, (Vol. 155, pp. 79-108). College Station, Texas: Ocean Drilling Program.

Prandtl, L. (1925). Bericht über Untersuchungen zur ausgebildeten Turbulenz. ZAMM-Journal of Applied Mathematics and Mechanics/Zeitschrift für Angewandte Mathematik und Mechanik, 5(2), 136-139.

Price, J. F., \& Sundermeyer, M. A. (1999). Stratified Ekman layers. Journal of Geophysical Research, 104(C9), $20467-20494$.

Pyles, D. R., Tomasso, M., \& Jennette, D. C. (2012). Flow processes and sedimentation associated with erosion and filling of sinuous submarine channels. Geology, 40, 143-146.

Rebesco, M., Hernández-Molina, F. J., Van Rooij, D., \& Wåhlin, A. (2014). Contourites and associated sediments controlled by deep-water circulation processes: State of the art and future considerations. Marine Geology, 352, 111-154.

Seim, H. E., \& Gregg, M. C. (1997). The importance of aspiration and channel curvature in producing strong vertical mixing over a sill. Journal of Geophysical Research, 102, 3451-3472.

Seim, K. S., \& Fer, I. (2011). Mixing in the stratified interface of the Faroe Bank Channel overflow: The role of transverse circulation and internal waves. Journal of Geophysical Research, 116, C07022. https://doi.org/10.1029/2010JC006805

Seminara, G. (2006). Meanders. Journal of Fluid Mechanics, 554, 271-297.

Sequeiros, O. E. (2012). Estimating turbidity current conditions from channel morphology: A Froude number approach. Journal of Geophysical Research, 117, C04003. https://doi.org/10.1029/2011JC007201

Serchi, G. F., Peakall, J., Ingham, D. B., \& Burns, A. D. (2011). A unifying computational fluid dynamics investigation on the river-like to river-reversed secondary circulation in submarine channel bends. Journal of Geophysical Research, 116, C06012. https://doi.org/10.1029/ 2010JC006361

Sherwin, T. (2010). Observations of the velocity profile of a fast and deep oceanic density current constrained in a gully. Journal of Geophysical Research, 115, C03013. https://doi.org/10.1029/2009JC005557

Sous, D., Sommeria, J., \& Boyer, D. (2013). Friction law and turbulent properties in a laboratory Ekman boundary layer. Physics of Fluids, 25(4), 046602.

Straub, K. M., Mohrig, D., Buttles, J., McElroy, B., \& Pirmez, C. (2011). Quantifying the influence of channel sinuosity on the depositional mechanics of channelized turbidity currents: A laboratory study. Marine and Petroleum Geology, 28, 744-760. https://doi.org/10.1016/j. marpetgeo.2010.05.014

Straub, K. M., Mohrig, D., McElroy, B., Buttles, J., \& Pirmez, C. (2008). Interactions between turbidity currents and topography in aggrading sinuous submarine channels: A laboratory study. Geological Society of America Bulletin, 120, 368-385. https://doi.org/10.1130/ B25983.1

Sumner, E. J., Peakall, J., Dorrell, R. M., Parsons, D. R., Darby, S. E., Wynn, R. B., et al. (2014). Driven around the bend: Spatial evolution and controls on the orientation of helical bend flow in a natural submarine gravity current. Journal of Geophysical Research, Oceans, 119 (2), 898-913. https://doi.org/10.1002/2013JC009008

Taylor, J. R., \& Sarkar, S. (2008). Stratification effects in a bottom Ekman layer. Journal of Physical Oceanography, 38(11), $2535-2555$.

Turner, J. S. (1973). Buoyancy effects in fluids, (p. 367). New York: Cambridge Univ. Press.

Umlauf, L., \& Arneborg, L. (2009a). Dynamics of rotating shallow gravity currents passing through a channel. Part I: Observation of transverse structure. Journal of Physical Oceanography, 39, 2385-2401. https://doi.org/10.1175/2009JPO4159.1

Umlauf, L., \& Arneborg, L. (2009b). Dynamics of rotating shallow gravity currents passing through a channel. Part II: Analysis. Journal of Physical Oceanography, 39, 2402-2416. https://doi.org/10.1175/2009JPO4164.1

Umlauf, L., Arneborg, L., Burchard, H., Fiekas, V., Lass, H. U., Mohrholz, V., \& Prandke, H. (2007). Transverse structure of turbulence in a rotating gravity current. Geophysical Research Letters, 34, L08601. https://doi.org/10.1029/2007GL029521 
Wåhlin, A. K., Darelius, E., Cenedese, C., \& Lane-Serff, G. F. (2008). Laboratory observations of enhanced entrainment in dense overflows in the presence of submarine canyons and ridges. Deep Sea Research Part I: Oceanographic Research Papers, 55, 737-750.

Wei, T., Peakall, J., Parsons, D. R., Chen, Z., Zhao, B., \& Best, J. (2013). Three-dimensional gravity-current flow within a subaqueous bend: Spatial evolution and force balance variations. Sedimentology, 60(7), 1668-1680.

Wells, M., Cenedese, C., \& Caulfield, C. P. (2010). The relationship between flux coefficient and entrainment ratio in density currents Journal of Physical Oceanography, 40, 2713-2727. https://doi.org/10.1175/2010JPO4225.1

Wells, M., \& Cossu, R. (2013). The possible role of Coriolis forces in structuring large-scale sinuous patterns of submarine channel-levee systems. Philosophical Transactions. Royal Society of London, 371, 20120366.

Wells, M. G. (2009). How Coriolis forces can limit the spatial extent of sediment deposition of a large-scale turbidity current. Sedimentary Geology, 218(1), 1-5.

Wynn, R. B., Cronin, B. T., \& Peakall, J. (2007). Sinuous deep-water channels: Genesis, geometry and architecture. Marine and Petroleum Geology, 24, 341-387.

Yang, S. -Q., Tan, S. K., \& Wang, X. -K. (2012). Mechanism of secondary currents in open channel flows. Journal of Geophysical Research, 117, F04014. https://doi.org/10.1029/2012JF002510

\section{References From the Supporting Information}

Barron, J., \& Ashton, C. (2005). The effect of temperature on conductivity measurement. TSP, 7(73), 1-5.

Cea, L., Puertas, J., \& Pena, L. (2007). Velocity measurements on highly turbulent free surface flow using ADV. Experiments in Fluids, 42, 333-348.

Chen, C. T., \& Millero, F. J. (1977). The use and misuse of pure water PVT properties for lake waters. Nature, $266(5604), 707$.

Franca, M. J., \& Lemmin, U. (2006). Eliminating velocity aliasing in acoustic Doppler velocity profiler data. Measurement Science and Technology, 17(2), 313-322.

McLelland, S. J., \& Nicholas, A. P. (2000). A new method for evaluating errors in high-frequency ADV measurements. Hydrological Processes, 14, 351-366.

Nortek, A. S. (2011). VECTRINO II profiling velocimeter user guide (Rev. C), Norway.

Strom, K. B., \& Papanicolaou, A. N. (2007). ADV Measurements around a cluster microform in a shallow mountain stream. Journal of Hydraulic Engineering, 133(12), 1379-1389.

Thomas, R. E., Schindfessel, L., McLelland, S. J., Creëlle, S., \& De Mulder, T. (2017). Bias in mean velocities and noise in variances and covariances measured using a multistatic acoustic profiler: The Nortek Vectrino Profiler. Measurement Science and Technology, 28, 0750302. https://doi.org/10.1088/1361-6501/aa7273

Voulgaris, G., \& Trowbridge, J. H. (1998). Evaluation of the acoustic Doppler velocimeter (ADV) for turbulence measurements. Journal of Atmospheric and Oceanic Technology, 15, 272-289.

Wahl, T. L. (2003). Discussion of "Despiking acoustic Doppler velocimeter data” by Derek G. Goring and Vladimir I. Nikora. Journal of Hydraulic Engineering, 129(6), 484-487.

Zedel, L., Hay, A. E., Cabrera, R., \& Lohrmann, A. (1996). Performance of a single-beam pulse-to-pulse coherent Doppler profiler. IEEE Journal of Oceanic Engineering, 21(3), 290-297. 